\title{
The conserved bromo-adjacent homology domain of yeast Orc1 functions in the selection of DNA replication origins within chromatin
}

\author{
Philipp Müller, ${ }^{1,2,8}$ Sookhee Park, ${ }^{1,2,8}$ Erika Shor, ${ }^{1,2,9}$ Dana J. Huebert, ${ }^{3}$ Christopher L. Warren, ${ }^{4,5}$ \\ Aseem Z. Ansari, ${ }^{4,5}$ Michael Weinreich, ${ }^{6}$ Matthew L. Eaton, ${ }^{7}$ David M. MacAlpine, ${ }^{7}$ \\ and Catherine A. Fox ${ }^{1,2,3,10}$ \\ ${ }^{1}$ Department of Biomolecular Chemistry, University of Wisconsin-Madison, Madison, Wisconsin 53706, USA; ${ }^{2}$ School of \\ Medicine and Public Health, University of Wisconsin-Madison, Madison, Wisconsin 53706, USA; ${ }^{3}$ Program in Cellular and \\ Molecular Biology, College of Agricultural and Life Sciences, University of Wisconsin-Madison, Madison, Wisconsin 53706, \\ USA; ${ }^{4}$ Department of Biochemistry, University of Wisconsin-Madison, Madison, Wisconsin 53706, USA; ${ }^{5}$ College of \\ Agricultural and Life Sciences, University of Wisconsin-Madison, Madison, Wisconsin 53706, USA; ${ }^{6}$ Laboratory for \\ Chromosome Replication, Van Andel Research Institute, Grand Rapids, Michigan 49503, USA; ${ }^{7}$ Pharmacology and Cancer \\ Biology, Duke University Medical Center, Durham, North Carolina 27710, USA
}

The origin recognition complex (ORC) binds to the specific positions on chromosomes that serve as DNA replication origins. Although ORC is conserved from yeast to humans, the DNA sequence elements that specify ORC binding are not. In particular, metazoan ORC shows no obvious DNA sequence specificity, whereas yeast ORC binds to a specific DNA sequence within all yeast origins. Thus, whereas chromatin must play an important role in metazoan ORC's ability to recognize origins, it is unclear whether chromatin plays a role in yeast ORC's recognition of origins. This study focused on the role of the conserved N-terminal bromo-adjacent homology domain of yeast Orc1 (Orc1BAH). Recent studies indicate that BAH domains are chromatin-binding modules. We show that the Orc1BAH domain was necessary for ORC's stable association with yeast chromosomes, and was physiologically relevant to DNA replication in vivo. This replication role was separable from the Orc1BAH domain's previously defined role in transcriptional silencing. Genome-wide analyses of ORC binding in ORC1 and orc1bahs cells revealed that the Orc1BAH domain contributed to ORC's association with most yeast origins, including a class of origins highly dependent on the Orc1BAH domain for ORC association (orc1bahs-sensitive origins). Orc1bahs-sensitive origins required the Orc1BAH domain for normal activity on chromosomes and plasmids, and were associated with a distinct local nucleosome structure. These data provide molecular insights into how the Orc1BAH domain contributes to ORC's selection of replication origins, as well as new tools for examining conserved mechanisms governing ORC's selection of origins within eukaryotic chromosomes.

[Keywords: ORC; origins; DNA replication; chromatin; yeast; BAH domains]

Supplemental material is available at http://www.genesdev.org.

Received January 15, 2010; revised version accepted May 11, 2010.

The first step in genome duplication for any organism is the appropriate selection of origins, the chromosomal sites where DNA replication begins. In eukaryotic cells, the origin recognition complex (ORC), an evolutionarily conserved heterohexamer, selects DNA replication origins by binding to specific positions on chromosomes (Bell

\footnotetext{
${ }^{8}$ These authors contributed equally to this work.

${ }^{9}$ Present address: Department of Molecular Biology, Princeton University, Princeton, NJ 08542.

${ }^{10}$ Corresponding author.

E-MAIL cfox@wisc.edu; FAX (608) 262-5253.

Article is online at http://www.genesdev.org/cgi/doi/10.1101/gad.1906410.
}

2002). During G1 phase of the cell cycle, ORC recruits additional factors to assemble the prereplication complex (pre-RC), culminating in the loading of the MCM replicative helicase (Diffley 2004; Stillman 2005). When cells enter S phase, phosphorylation of pre-RC components by S-phase kinases triggers the recruitment of additional proteins and the unwinding of DNA replication origins (Stillman 2005; Sclafani and Holzen 2007). The proteins, including ORC, and the steps in assembly and activation of origin complexes are fairly well conserved from budding yeast to humans (Bell and Dutta 2002). However, it is unclear whether mechanisms involved in ORC's selection of 
origins are conserved, since origins themselves, in terms of DNA sequence, are not conserved among eukaryotic organisms (Mechali 2001; Gilbert 2004; Cvetic and Walter 2005; Aladjem 2007; Rampakakis et al. 2009). This study sought to identify conserved features of ORC's selection of DNA replication origins by focusing on the role of the ORC bromo-adjacent homology (BAH) domain in budding yeast.

The mechanism by which ORC selects a chromosomal replication origin is best understood in yeast. Yeast origins, named ARSs (autonomous replicating sequences) for their ability to endow autonomous replication to plasmids, contain an ORC-binding site, a bipartite DNA sequence containing a conserved 15-base-pair (bp) A-element, and a smaller, less conserved, B1-element (Rao and Stillman 1995; Xu et al. 2006; Chang et al. 2008). When yeast ORC binds to its site, it protects $\sim 35$ bp of DNA that includes these defined elements (Lee and Bell 1997). The ORCbinding site is absolutely necessary but not sufficient for origin function (Marahrens and Stillman 1992; Newlon and Theis 1993). Although all yeast origins contain the conserved ORC-binding site, some evidence indicates that yeast ORC also relies on other mechanisms to select replication origins. For example, the yeast genome contains thousands of matches to an ORC-binding consensus site, yet only a small fraction of these bind ORC in vivo (Nieduszynski et al. 2007). In addition, several bona fide yeast replication origins bind ORC weakly in vitro yet function robustly in vivo (Palacios DeBeer et al. 2003). These observations suggest a potential similarity in origin selection mechanisms between yeast and metazoans where the few characterized origins lack DNA sequence conservation and ORC shows low DNA sequence specificity in vitro (Vashee et al. 2003; Remus et al. 2004).

Orc1 orthologs from yeast to humans contain a BAH domain at their N termini (Bell et al. 1995; Gavin et al. 1995). BAH domains are discrete protein domains of $\sim 200$ amino acids that can be identified in many different nuclear proteins throughout the eukaryotic kingdom, suggesting a fundamental role in chromatin (Callebaut et al. 1999; Goodwin and Nicolas 2001). Recent biochemical studies indicate that the highly similar BAH domains of yeast Orc1 and Sir3 bind chromatin, and experiments with purified components reveal that the Sir3BAH domain binds directly to nucleosomes (Onishi et al. 2007). In human cells, the BAH domain of Orc1 contributes to ORC's role in chromosome binding and the function of a viral replication origin (Noguchi et al. 2006). However, no evidence supports a role for the Orc1BAH domain in yeast DNA replication. In fact, in contrast to deletions in any of the six ORC genes, a deletion of the coding region for the Orc1BAH domain to create an orc1bahs mutation has no effect on yeast cell viability, S-phase progression, or the rate at which a plasmid is lost from dividing yeast cells (Bell et al. 1995). Instead, the only documented role for the yeast Orc1BAH domain is in the nonessential process of transcriptional silencing, a form of position-dependent chromatin-mediated repression akin to heterochromatin that occurs at the silent mating type loci $H M R$ and $H M L$ (Fox and McConnell 2005).

Eukaryotic chromosomes contain many more origins than necessary for their efficient replication, and no single origin is essential. Thus, it is conceivable that the Orc1BAH domain contributes to ORC's role in selecting a subset of yeast origins without causing defects in cell growth or viability usually associated with global impairment of DNA replication. Therefore, we directly assessed the role of the N-terminal BAH domain of Orc1 in DNA replication by comparing wild-type ORC1 and mutant orc1bahs cells. Biochemical analyses revealed that the Orc1BAH domain was important for ORC's stable association with yeast chromatin, and genetic analyses indicated that the Orc1BAH domain contributed to the essential process of DNA replication via mechanisms distinct from its established role in transcriptional silencing. Chromatin immunoprecipitation (ChIP) with Orcl antibodies followed by microarray analyses (ChIP-on-chip) revealed that the Orc1BAH domain contributed to ORC binding at most yeast origins, and contributed quite substantially to a group of origins termed orc1bahs-sensitive origins. A unique ORC DNA-binding consensus site and nucleosome organization distinguished the orc1bahs-sensitive origins. Thus, yeast ORC selects origins through a combination of ORC-DNA and ORC-chromatin interactions, and the extent to which one type of interaction contributes to origin selection varies with individual origins.

\section{Results}

The Orc1BAH domain contributed to yeast ORC's association with chromatin

To determine whether proteins other than Sirl interacted with ORC in an Orc1BAH-dependent manner, anti-Orc1directed immunoprecipitation (IP) was performed on nuclear extracts from ORC1 wild-type or orc1bahs mutant cells. Nuclear extracts were prepared and the chromatin was digested with MNase to generate primarily mono-, di-, and trinucleosomes (Supplemental Fig. S1). Relative levels of proteins recovered in the two different immunoprecipitates were determined using isobaric tags for relative and absolute quantitation (iTRAQ) (Applied Biosystems) (Fig. 1A; Ross et al. 2004). In a typical iTRAQ experiment, the peptides generated from different samples are labeled with different mass markers, such that individual peptides can be quantitatively compared between two samples that are analyzed simultaneously by mass spectrometry (TOF-TOF LC/MALDI MS/MS). Thus, an orc1bahs/ORC1 iTRAQ ratio of 1.0 meant that a peptide was present at equal levels in the two different IPs, whereas a ratio of $<1.0$ meant that a peptide was present at higher levels in the IP from ORC1 cell extracts compared with orc1bahs mutant cell extracts.

The ORC subunits and the core histones comprised the most abundant peptides recovered in the IP (Fig. 1B; Supplemental Table S1). In addition, in contrast to the majority of ORC and non-ORC polypeptides that were recovered similarly in the IPs from ORC1 and orc1bahs 
Müller et al.
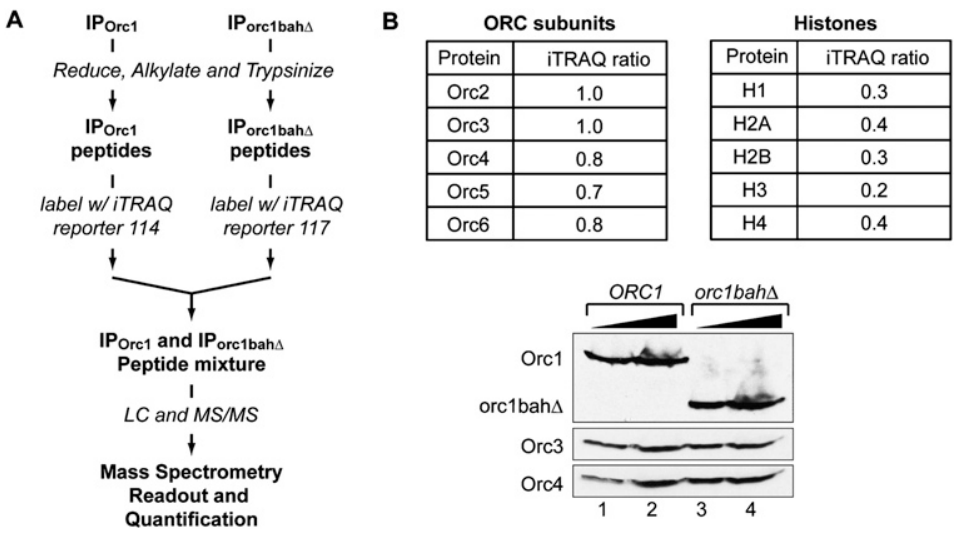

C

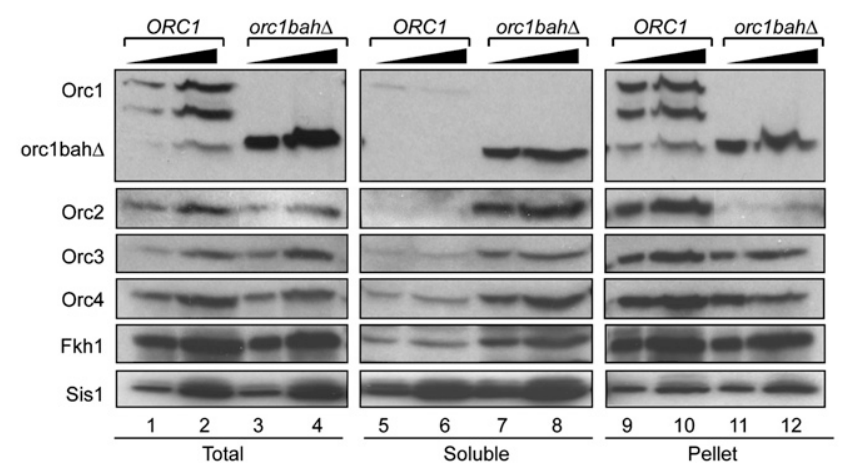

Figure 1. The Orc1BAH domain contributed to ORC's association with chromatin in yeast. $(A)$ Outline of protocol used to compare immunopurified ORC from chromatin extracts prepared from ORC1 wild-type or orc 1 bahs mutant cells. See Supplemental Figure S1 for more detail. (B) The top panel on the left reports average iTRAQ ratios calculated for each ORC subunit except Orc1. (The Applied Biosystems algorithm could not determine the average iTRAQ ratio for the Orc1BAH domain and the remaining portion of Orc 1, as it uses wild-type $S$. cerevisiae protein database as reference.) The top panel on the right reports average iTRAQ ratios calculated for each of the core histones and yeast histone H1. The average iTRAQ orc1bahs/ORC1 ratio for individual ORC subunits (average of all of the orc $1 b a h \Delta /$ ORC iTRAQ peptide ratios that comprised each polypeptide, determined by an Applied Biosystems algorithm) was close to 1.0, indicating that an anti-Orc1 IP recovered similar levels of ORC from wildtype ORC1 and orc1bahs mutant cells. In contrast, the average iTRAQ ratio for each of the core histones was $\leq 0.4$, indicating that histones were recovered less efficiently from orc1bahs mutant compared with ORC1 cells. The bottom panel is a protein immunoblot of Orc1, Orc3, and Orc4 subunits from ORC1 wild-type and orc1bahs mutant cell extracts. $\mathrm{A}_{600}$ cell equivalents of 0.25 (lanes 1,3) and 0.5 (lanes 2,4) were loaded. $(C)$ Crude extracts from ORC1 wild-type or orc1bahs mutant cells (lanes 1-4, total) were fractionated by centrifugation into soluble (lanes 5-8, soluble) and chromatin-containing pellet (lanes 9-12, pellet) fractions. $\mathrm{A}_{600}$ cell equivalents of 0.38 O.D. and 0.75 O.D. of total extracts and soluble and pellet fractions were analyzed by protein immunoblotting for the indicated proteins. Orc1 from wild-type cells produced three detectable protein fragments in these experiments, only one of which corresponded to predicted full-length Orc1 protein, presumably because of Orcl protein degradation. The transcription factor Fkh1 was also analyzed in this experiment, with polyclonal antibodies against Fkh1 (Casey et al. 2008). The cytosolic chaperone Sis1 was analyzed with polyclonal antibodies against Sis1 (Yan and Craig 1999).

cells, each of the histones was recovered less well from orc1bahs compared with ORC1 cells. Specifically, the ORC subunits were recovered at similar levels from ORC1 and orc1bahs mutant cells (ORC subunits were recovered at $70 \%-100 \%$ efficiency from orc 1 bahs relative to ORC1 cells). However, the histones were recovered poorly from orc $1 \mathrm{bah} \Delta$ mutant compared with ORC1 cells $(20 \%-40 \%$ efficiency in orc 1 bah $\Delta$ mutant compared with wild-type ORC1 cells) (Fig. 1B). Thus, while the orc1bahs mutation did not affect the integrity of ORC, it reduced ORC's association with histones within a nuclear extract of fragmented chromatin.

To test whether the Orc1BAH domain contributed to ORC's association with intact chromosomes, whole-cell extracts were fractionated by centrifugation into chromosome-containing (pellet) and chromosome-free (soluble) fractions (Liang and Stillman 1997), and each fraction was examined for individual ORC subunits by protein immunoblotting (Fig. 1C). The total cell extracts from ORC1 and orc1bahs cells produced similar levels of each ORC subunit, as expected, although we note that Orc1 from wild-type cells generated three detectable proteins of different sizes in these experiments, presumably due to protein instability. The effect was to make the mutant orclbah $\Delta$ protein appear more abundant relative to wild- type Orc1 in these experiments compared with others, since a single band represented the mutant protein. Nevertheless, the other subunits were expressed at similar levels in ORC1 and orc1bahs cells (Fig. 1C, lanes 1-4). In extracts from ORC1 cells, individual ORC subunits were enriched in the pellet fraction relative to the soluble fraction, indicating that yeast ORC associated stably with chromatin, as expected (Fig. 1C, cf. lanes 5,6 and 9,10; Liang and Stillman 1997). In contrast, in extracts from orc1bahs cells, ORC subunits were equally if not more enriched in the soluble fraction compared with the pellet fraction (Fig. 1C, cf. lanes 7,8 and 11,12). We note that the Orc2 subunit seemed particularly enriched in the soluble fraction and depleted from the pellet fraction in orc $1 \mathrm{bahs}$ cells compared with the other subunits. This effect was observed in two independent experiments, and we do not have an explanation for this unexpected result; although, since Orc2 was recovered well in the iTRAQ experiment (Fig. 1B), it is possible that the effect in this experiment was a consequence of the sensitivity of the particular anti-Orc2 antibody used in immunoblotting. The cytosolic HSP40 cochaperone protein Sis1 was distributed similarly between the pellet and soluble fractions generated from ORC1 and orc1bahs extracts, indicating that the differences in fractionation were not observed for 
every protein in the extract. However, the transcription factor Fkh1 was enriched in the soluble fraction derived from orc1bahs compared with ORC1 cell extracts. These data raise the possibility that the orc1bahs mutation modulated the association of other DNA-binding factors with chromosomes, perhaps because a reduction in ORC occupancy at ARSs alters local nucleosome structure, thus affecting a significant fraction of the genome (Eaton et al. 2010). Taken together with the iTRAQ data, these results provided evidence that the yeast Orc1BAH domain contributed to ORC's association with yeast chromatin.

\section{The Orc1BAH domain contributed to ORC's} association with most origins

The canonical yeast ORC-binding site present in yeast origins contains a conserved A-element and a B1-element (Rao and Stillman 1995; Lee and Bell 1997). However, variations in the A- and B1-elements can affect ORC's affinity for origin DNA in vitro, indicating that not all origins provide for equally robust core ORC-DNA interactions (Palacios DeBeer et al. 2003). Based on the above data (Fig. 1), and recent data providing evidence that the $\mathrm{BAH}$ domains of Orc1 and Sir3 bind chromatin /Cubizolles et al. 2006; Onishi et al. 2007; Buchberger et al. 2008; Sampath et al. 2009), a reasonable postulate was that dependence of an origin on the Orc1BAH domain for ORC binding might vary between origins due to differences in core ORCbinding sites and/or surrounding chromatin. To test this possibility, ORC binding was compared in ORC1 and orc1bahs mutant cells using ChIP with $\alpha$-Orc1 antibodies followed by microarray analyses of the yeast genome (ChIP-on-chip).

For the array data generated from ORC1 ChIP DNA (ORC1 array), peaks were identified using ChiPotle (Buck et al. 2005) with a $P$-value cutoff of $<10^{-20}$ (512 peaks). From the resulting list, peaks with a $P$-value cutoff of $<10^{-30}$ (398 peaks) were included for further analyses (Fig. 2A; Supplemental Table S2). This $P$-value cutoff was chosen based on visual inspection of the raw data provided by Nimblegen, and maximal overlaps between the data generated in the arrays in this study and other yeast genome origin data sets. The ORC peaks identified in the ORC1 array showed substantial overlap with the likely and confirmed ARSs listed in the yeast OriDB (http:// www.oridb.org; Nieduszynski et al. 2007) and ORC and
A

398 individual ORC peaks identified using ChIPotle

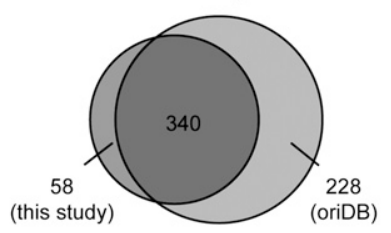

vs likely and confirmed ARS from oriDB (568 sites)

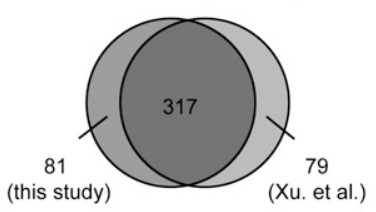

vs ORC and ORC/MCM2 peaks from Xu et al. (396 sites)
B

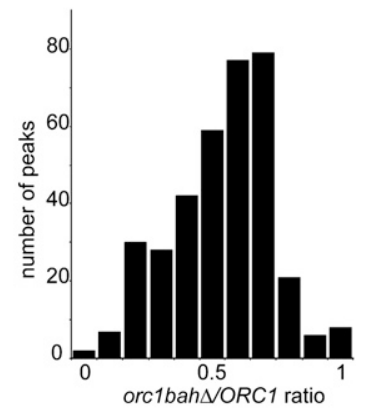

Figure 2. The Orc1BAH domain contributed differentially to ORC's association with ARSs. ChIP-on-chip experiments were performed with anti-Orcl antibodies from ORC1 and orc1bahs cells. The chips used were customdesigned high-density tiled arrays from Nimblegen (Shor et al. 2009). (A) Overlap between the peaks identified in the ORC1 array with a $P$-value cutoff $<10^{-30}$ in this study with the likely and confirmed ARSs listed in OriDB (568 sites) (Nieduszynski et al. 2007) (top), and the ORC and ORC/ Mcm2 peaks identified by Aparicio and colleagues (Xu et al. 2006) (bottom). (B) Graph of number of peaks identified in the ORC1 wild-type array ( $Y$-axis) against their orc1bahs/ORC1 ratio ( $X$-axis). (C) Peaks from microarray experiments for representative ARSs.

C

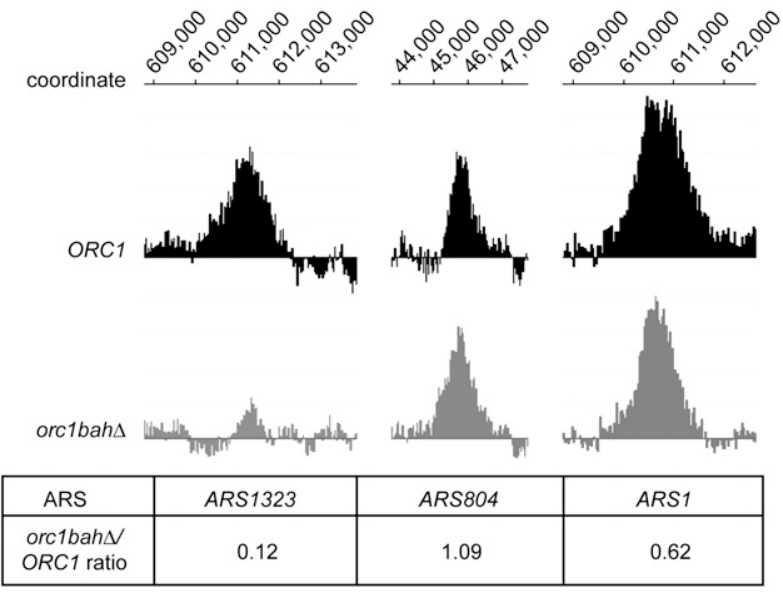


Müller et al.

ORC/MCM sites identified by Aparicio and colleagues (Fig. 2A; Xu et al. 2006). A similar number of peaks was identified in the orc1bahs array, but most of these were smaller (P. Müller, unpubl.), suggesting that the Orc1BAH domain affected ORC binding to most origins, as expected based on the data presented in Figure 1. The average size of DNA fragments was similar in ORC1 and orc1bahs cells (on average, $\sim 250-500$ bp) (P Müller, unpubl.). Therefore, comparing the area of an individual peak in wild-type and mutant strains should reflect differences in the efficiency of IP of the corresponding DNA fragments. Accordingly, we interpreted a reduction in the area of an ORC-associated peak from orc1bahs relative to ORC1 and cells as a reduction in ORC occupancy. Thus, for every peak identified in the ORC1 array, the ratio of its area in the orc1bahs array to that in the ORC1 array (orc1bah $/$ ORC1 ratio, $X$-axis) was determined and plotted against the number of peaks (Y-axis) (Fig. 2B; see also Supplemental Table S2). This plot revealed that ORC's association with most origins was reduced in orc1bahs mutant cells. For peaks that showed reductions in area, the individual peak in orc1bahs cells was both narrower and lower compared with the ORC1 peak (Fig. 2C).

Although the Orc1BAH domain influenced ORC's association with the vast majority of origins, it was evident from the graph in Figure $2 \mathrm{~B}$ that a subset of ARSs was particularly affected for ORC association by the orc1bahs mutation, whereas another subset was relatively unaffected. To understand how the Orc1BAH domain helped ORC bind to origins, these two extreme classes of origins were analyzed further. Specifically, confirmed and likely origins (http://www.oridb.org) associated with peaks that were either substantially reduced in size in the orc1bahs array compared with the ORC1 array (orc1bah $\Delta / O R C 1$ ratio $<0.33$; orc 1 bah $\Delta$-sensitive) or relatively unaffected (orc1bah $\Delta /$ ORC1 ratio $>0.80$; orc1bah $\Delta$-resistant) were chosen. Representative examples of an orc1bahs-sensitive peak (ARS1323), an orc1bahs-resistant peak (ARS804), and the moderately affected peak associated with $A R S 1$ are shown in Figure 2C.

Most peaks in either extreme category were associated with confirmed or likely ARSs as listed in the OriDB (http://www.oridb.org), although peaks in the orc1bahsresistant category had a higher probability of being associated with a confirmed or likely ARS $172 \%$ of sensitive compared with $84 \%$ of resistant peaks were associated with a confirmed or likely ARS). On average, the peak areas for ARSs in either extreme category were smaller than the peak areas for the majority of ARSs in the array (Supplemental Fig. S2). ARS-specific ChIPs using quantitative real-time PCR (qPCR) were performed for an orc1bahs-sensitive ARS (ARS1323) and an orc1bahsresistant ARS (ARS804) (Fig. 3A,B). Regions that immediately flanked the ARS1323 ORC-binding site (detected by

A
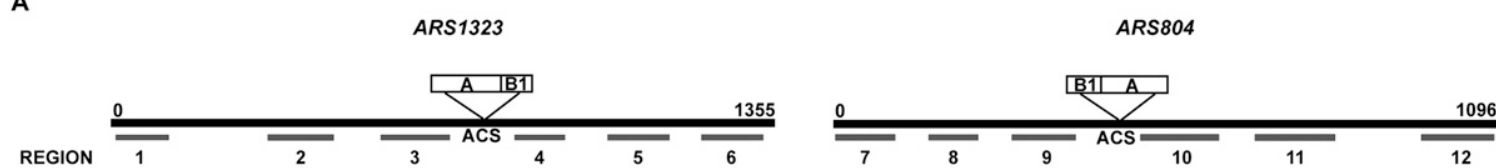

B

ORC-ChIP
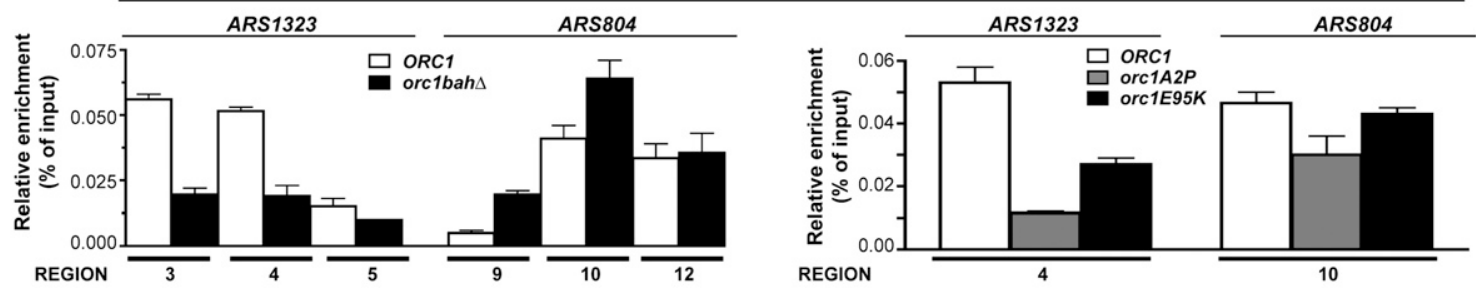

C

MCM-ChIP
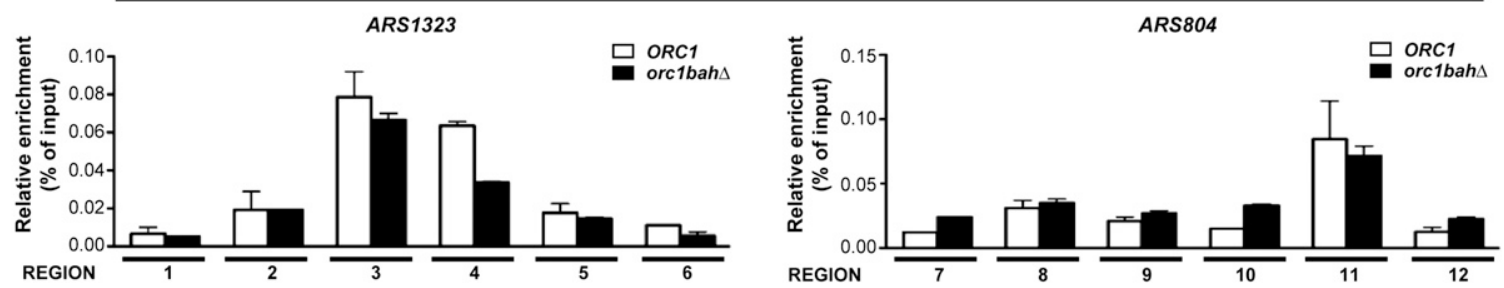

Figure 3. Mutation of Orc1BAH specifically affects ORC and MCM association with an orc1bah $\Delta$-sensitive ARS. qPCR of ChIP experiments was used to analyze ORC and MCM association with ARS1323 and ARS804. (A) Diagram of ARS1323 and ARS804. Numbers below the gray lines refer to the $\sim 100$-bp regions detected by primers described in Supplemental Table S4. (B) Orc1-directed ChIP of these ARSs show that either deletion or point mutation (A2P or E95K) of Orc1BAH reduced ORC association with the orc1bahs-sensitive ARS1323 but not the orc1bahs-resistant ARS804. (C) MCM-directed ChIPs show that deletion of Orc1BAH reduced MCM association with a region near ARS1323 (region 4) but not ARS804. 
PCR amplification of regions 3 and 4) (Fig. 3A) were recovered threefold less efficiently from orc1bahs compared with ORC1 cells. In contrast, the analogous regions in ARS804 were actually recovered more efficiently from orc1bahs compared with ORC1 cells. These ARS-specific ChIPs mirrored the array data for these ARSs (Fig. 2C). In summary, the Orc1BAH domain contributed to ORC's association with most ARSs in the yeast genome, but two extreme classes of ARSs could be identified: one class in which the Orc1BAH domain contributed substantially to ORC association (orc1bahs-sensitive), and one class in which it did not (orc1bahs-resistant).

Orc1 amino acids conserved between the Orc1BAH and Sir3BAH domains contributed to ORC association with an orclbah $\Delta$-sensitive $A R S$

The Sir3BAH and Orc1BAH domains are significantly similar at both the sequence and structural levels (Bell et al. 1995; Zhang et al. 2002; Connelly et al. 2006; Hou et al. 2006), and both domains bind nucleosomes from crude chromatin extracts (Onishi et al. 2007). In vitro studies of Sir3BAH-chromatin interactions indicate that several amino acids conserved between OrclBAH and Sir3BAH domains contribute to the ability of Sir3BAH to bind chromatin (Buchberger et al. 2008). If similar interactions contributed to ORC's association with ARSs, then the analogous residues in the Orc1BAH domain should contribute to ORC's association with an orc1bahs-sensitive ARS. Therefore, mutations encoding two different amino acid substitutions, A2P and E95K, were engineered into the chromosomal ORC1 locus. The A2P mutation prevents N-terminal acetylation of Orc1, and is important for Sir3BAH domain's interactions with chromatin. The E95K substitution is analogous to a mutation in the Sir3BAH domain that abolished its ability to bind nucleosomes in vitro (Buchberger et al. 2008). Both mutants produced similar levels of Orc1 (S Park and CA Fox, unpubl.), and both reduced ORC's association with the orc1bah $\Delta$-sensitive ARS ARS1323 by fivefold and twofold, respectively, as measured by qPCR (Fig. 3B). In contrast, the A2P and E95K mutant proteins produced relatively minor defects in ORC's association with an orc1bahs-resistant ARS, ARS804, reducing ORC binding by 1.6 -fold and 1.1 -fold, respectively. However, in contrast to the orclbah $\Delta$ mutant, neither mutant caused an enhancement of ORC association with ARS804. Several explanations are possible, including that an ORC lacking an Orc1BAH domain is cross-linked more efficiently to ARS804 during ChIP. Nevertheless, these data provided evidence that regions of the Orc1BAH domain expected to contribute to nucleosome binding in vitro also contributed to ORC's association with specific ARSs in vivo, particularly an orc1bahs-sensitive ARS.

The Orc1BAH domain contributed to MCM association with an orclbah $\Delta$-sensitive ARS

An established role for ORC in origin firing is to assemble the pre-RC, which culminates in the loading of the MCM helicase at origins. Therefore, a defect in ORC binding at a particular ARS would be predicted to reduce the association of MCM with that ARS, although it is important to note that the level of ORC occupancy needed for a given level of MCM association is unknown. To test whether the Orc1BAH domain contributed differentially to MCM association with ARS1323 and ARS804, antiMcm2 ChIPs were performed (Fig. 3C). Since qPCR was used in the detection step in this experiment, and it was unclear which region of these ARSs would show maximal MCM association, several different primer pairs that spanned the ARS1323 and ARS804 chromosomal regions were used (Fig. 3A). The most significant MCM binding at ARS1323 was detected at regions 3 and 4, and a 1.7-fold reduction in MCM association was observed at region 4 in orc1bahs compared with ORC1 cells (Fig. 3C). For ARS804, significant MCM association was detected only at region 11, and no significant reduction in MCM association was detected in orc1bahs mutant cells at this region. These data provided evidence that the orc1bahs mutation reduced MCM association with an orc1bahssensitive ARS.

Orc1bahs-and orc2-1-sensitive origins were not highly related

The Orc1BAH domain contributed to ORC's stability on chromosomes and association with a large number of ARSs (Fig. 2B). To determine whether orc1bahs-sensitive origins might simply reflect a class of lower-affinity ORC target sites, we compared ORC binding at ARSs in orc1bahs and orc2-1 mutant cells. Orc2-1 is a temperature-sensitive allele of the ORC2 gene that partially compromises ORC even at permissive growth temperatures (Fox et al. 1995). The molecular defect caused by orc2-1 is to reduce the levels of Orc2 subunit and the stability of ORC through purification (Bell et al. 1993; Shimada et al. 2002). The concentration of functional ORC is reduced in orc2-1 mutant cells such that only higheraffinity ORC sites remain fully occupied by ORC in these cells (Palacios DeBeer et al. 2003; Shor et al. 2009). For example, $H M R$-E, an element with a known high-affinity ORC site, remains fully occupied by ORC as measured by ChIP in orc2-1 mutant cells, whereas ARS1, an ARS with a lower-affinity ORC site, does not (Shor et al. 2009). A ChIP-on-chip experiment with wild-type and orc2-1 mutant cells was performed. Detailed analyses of these data are presented elsewhere (Shor et al. 2009). For this study, the relatedness of orc1bahs-and orc2-1-sensitive ARSs in terms of ORC association was determined. Each ARSassociated peak identified in the ORC1 array was positioned at its orc1bahs/ORC1 ratio on the $Y$-axis and its corresponding orc2-1/ORC2 ratio on the $X$-axis (Fig. 4A). If orc1bahs and orc2-1 sensitivity reflected a common defect in ORC-ARS recognition, there should be a strong positive correlation between orc1bahs and orc2-1 sensitivities for all origins. But instead, this plot revealed that orc1bahsand orc2-1-sensitive ARSs were not correlated $\left(R^{2}=\right.$ $0.0316)$. These data provided evidence that the orc1bahs and orc2-1 mutations affected different features of the ORC-ARS interface that stabilized ORC at origins. 
Müller et al.

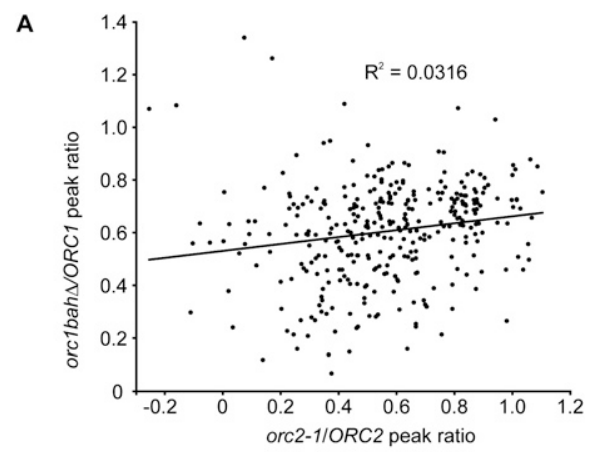

B

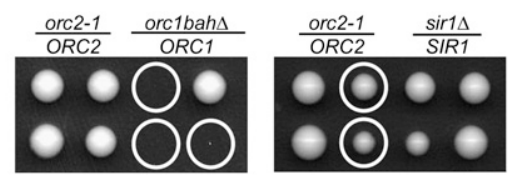

Figure 4. The orc1bahs and orc2-1 mutations affected different features of origin recognition by ORC. $(A)$ For all the likely and confirmed ARSs identified in the ORC1 array, their orc1bahs/ ORC1 peak size ratios were determined ( $Y$-axis) (this study) and plotted against their corresponding orc2-1/ORC2 peak size ratio (Shor et al. 2009). (B) Representative tetrads from crosses between orc2-1 and orc1bahs cells and orc2-1 and sir1s cells. Viable haploid cells containing both the orc2-1 (measured by temperature-sensitive growth) and orc1bahs (measured by PCR) alleles were not recovered from 10-20 tetrads examined from three independent crosses. Tetrads are shown from a cross between

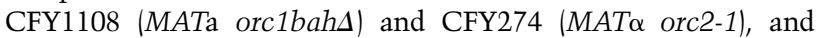
between CFY274 (MAT $\alpha$ orc2-1) and CFY430 (MATa sir14::LEU2). The orc2-1 allele was identified by temperature-sensitive growth and the orc $1 b a h \Delta$ allele was identified by PCR. The sir $1 \Delta:: L E U 2$ allele was identified by growth on minimal medium lacking leucine.

The Orc1BAH domain was essential for viability in orc2-1 mutant cells

Since the data discussed above (Fig. 4A) suggested that orc2-1 and orc1bahs contributed to independent features of ORC's recognition of ARSs, we suspected that the two mutations together might reduce origin activity below a minimal threshold required for robust cell growth. To test this possibility, orc1bahs and orc2-1 cells were crossed to generate haploids harboring both the orc2-1 and orc1bahs mutant alleles.

Several independent crosses revealed that the orc2-1 and orc1bahs alleles were synthetically lethal (Fig. 4B). Examination of spores from 10 independent tetrads indicated that viable haploid cells containing both of these alleles were not recovered.

The molecularly documented role for the yeast Orc1BAH domain is to bind Sirl and function in $H M R$ and $H M L$ silencing (for review, see Fox and McConnell 2005). To test whether this role was distinct from the role of the Orc1BAH domain in helping ORC bind to ARSs, a cross was performed between orc2-1 and sir1s cells. The tetrads produced from this cross had four viable spores, including haploids containing both the orc2-1 and sir1s alleles (Fig. 4B). Therefore, the Orc1BAH domain was required for yeast cell viability when ORC function was compromised by the orc2-1 mutation, and this role was independent of SIR1. These data also provided evidence that the role of the Orc1BAH domain relevant to DNA replication was separable from its role in transcriptional silencing.

\section{The Orc1BAH domain modulated replication initiation}

The data discussed above provided evidence that the Orc1BAH domain contributed to ORC's stable association with ARSs in vivo. However, it is unclear how stably ORC must associate with any particular ARS to assemble a pre-RC and function in chromosomal origin firing. In particular, it was possible that an orc1bahs-sensitive ARS might be unaffected in terms of origin firing, since some ORCs clearly remained bound to these sites even in orc1bahs mutant cells (Fig. 2B,C) and an orc1bahssensitive ARS still associated with MCM in orc1bahs cells, although MCM levels were reduced (Fig. 3C). Therefore, the efficiency of origin firing for a few orc1bahsresistant and orc1bah $\Delta$-sensitive ARSs and ARS1, as a benchmark, was measured by two-dimensional (2D) origin mapping and plasmid loss rate assays in ORC1 and orc1bahs cells (Fig. 5; Supplemental Table S3).

The $2 \mathrm{D}$ origin mapping experiments revealed that orc1bahs sensitivity or resistance was not an indicator of origin efficiency in wild-type ORC1 cells. Specifically, based on the ratio of bubble intermediates to small forks, both ARS classes contained origins that were efficient (e.g., ARS804 [orc1bahA-resistant] and ARS1323 [orc1bahssensitive]) and inefficient (e.g., ARS1626 [orc1bahsresistant] and ARS446 [orc1bahs-sensitive]) in ORC1 cells. The orc1bahs-resistant origins ARS1626, ARS804, and $A R S 716$ fired with similar efficiencies in ORC1 and orc1bahs cells (Fig. 5A), consistent with the ChIP-on-chip data that ORC binding was not reduced at these ARSs in orc1bahs cells (Fig. 2B; Supplemental Table S2). In contrast, the orc1bahs-sensitive origin ARS1323 exhibited reduced origin firing in orc1bahs cells compared with ORC1 cells, with the levels of bubble intermediates reduced and the levels of small forks enhanced. These data were consistent with a substantial reduction of ORC binding at ARS1323 caused by the orc1bahs mutation (orc1bahs/ORC1 ratio of 0.12) (Fig. 2C). For several other orc1bahs-sensitive ARSs, the 2D origin mapping data were less clear in part because recovery of the DNA fragments that included these origins was consistently lower in orc1bahs compared with ORC1 cells. In particular, it was difficult to discern an obvious increase in small forks for orc1bahA-sensitive ARS1211, ARS446, ARS432.5, and ARS822. Nevertheless, with the exception of ARS1211, the origin bubble intermediates appeared reduced in quantity relative to replication forks for these ARSs in orc1bahs compared with ORC1 cells at comparable exposures. In addition, there was another difference that was initially most obvious at ARS1323. Specifically, the origin bubble intermediates for ARS1323 in orc1bahs cells spanned the range of possible bubble sizes, from small 
A
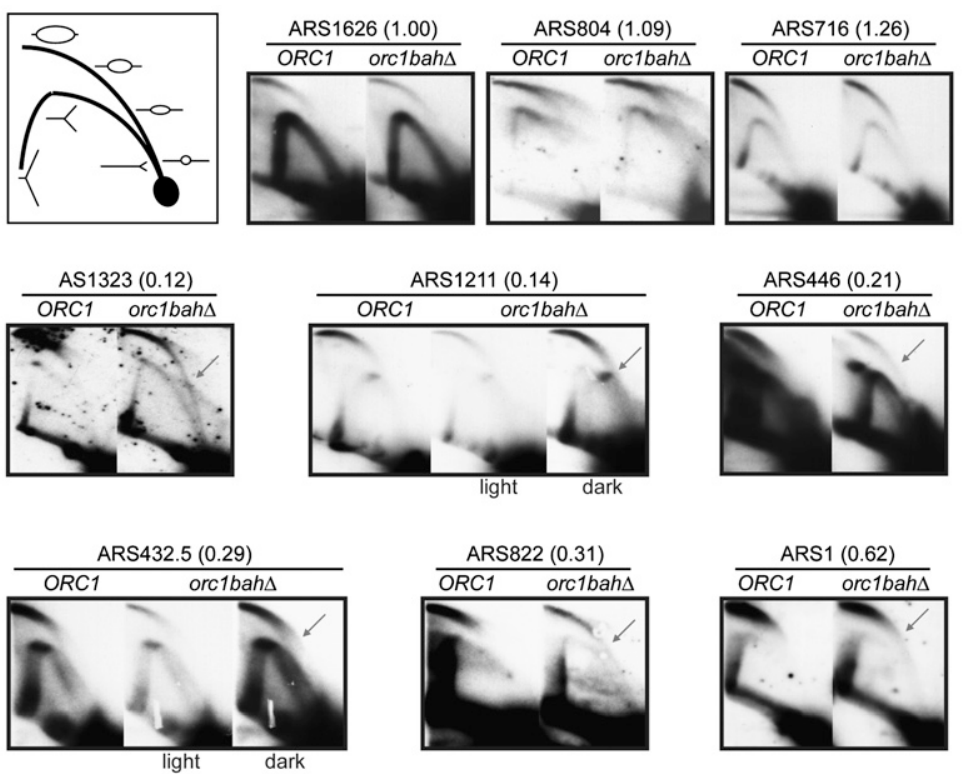

B

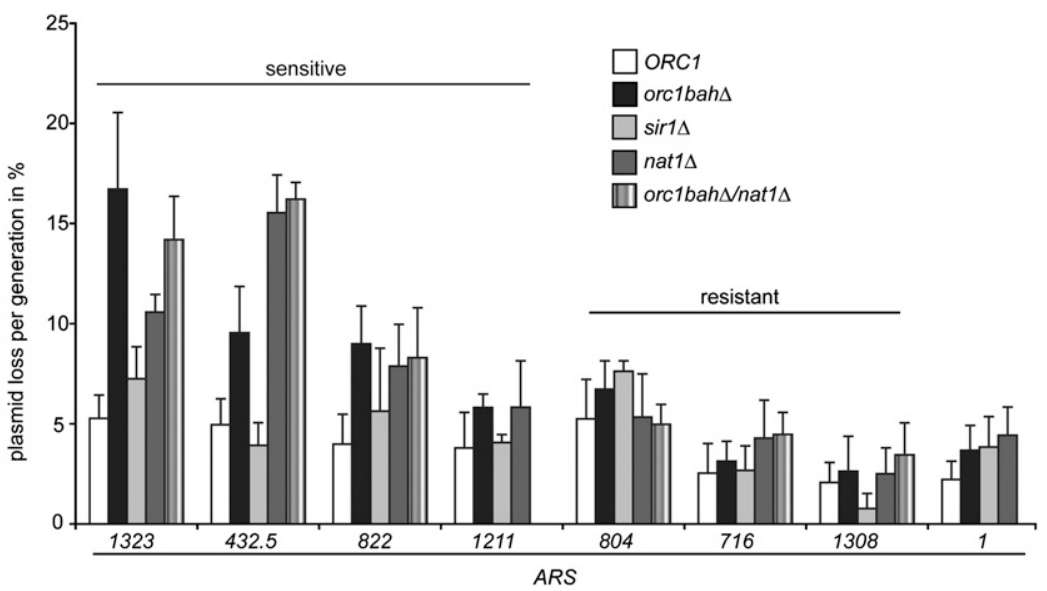

Yeast Orc1BAH domain in chromosome replication

Figure 5. The Orc1BAH domain modulated replication initiation. (A) Two-dimensional origin mapping on the indicated chromosomal origins in ORC1 (CFY145) and orc1bahs (CFY1108) cells. The orc1bahs/ORC1 peak size ratio as determined from the ChIP-on-chip data is indicated parenthetically next to the ARS name. The arrows indicate examples in which origin bubble intermediates show increased heterogeneity in orc $1 \mathrm{bah} \Delta$ cells. Two different exposures of film are shown for the 2D origin mapping experiments for ARS1211 and ARS432.5 in orc1bahs cells. Details about restriction fragments and radioactive probes used for these experiments are included in Supplemental Table S3. (B) Results from plasmid loss rate assays for the indicated strains and various ARS-containing plasmids: ORC1 (CFY145), orc1bahs (CFY1108), sir1s (CFY345), nat1s (CFY2922), and orc1bahs nat1s (CFY3086). to large (see arrow in Fig. 5A), whereas, in ORC1, cells the bubble signal was more homogenous and mostly large, indicative of an enrichment of large bubbles relative to smaller bubbles for this ARS in wild-type cells. A similar increase in the heterogeneity of origin bubble intermediates could be observed on careful examination of the other sensitive ARSs (Fig. 5A, see arrows). This heterogeneity in sizes of bubble intermediates was less obvious for the orc1bahs-resistant ARSs, suggesting this feature was relevant to ORC occupancy differences associated with the orc1bahs mutation. Finally, ARS1, which was moderately affected for ORC binding by the orc1bahs mutation (orc1bah $/$ ORC1 ratio $=0.62$ ) (Fig. 2C), was also examined. Although no increase in the level of small forks could be detected, it was clear that the intensity and homogeneity of large bubble intermediates relative to smaller bubble intermediates was reduced in orc1bahs compared with ORC1 cells. In summary, the 2D origin mapping experiments revealed that the orc1bahs mutation affected chromosomal origin firing patterns, and orc1bahs-sensitive ARSs were affected more significantly than orc1bahsresistant ARSs.
If relatively local as opposed to long-range features of the chromosomal regions containing these ARSs were responsible for their differences in Orc1BAH dependency, then these differences should be recapitulated in a plasmid loss assay. Furthermore, plasmid loss assays could provide a level of sensitivity in detecting quantitative defects in origin function that could not be obtained by $2 \mathrm{D}$ origin mapping. Therefore, $\sim 1-\mathrm{kb}$ regions centered around the predicted A-B1-element (OriDB) of three different orc1bahs-sensitive origins and three different orc1bahs-resistant origins were cloned into a plasmid that lacked an ARS, and plasmid loss rates were determined in ORC1 and orc1bahs cells (Fig. 5B). ARS1 was also examined in these experiments. Three orc1bahssensitive ARSs (ARS1323, ARS432.5, and ARS822) were lost at a twofold to threefold higher rate per cell generation in orc1bahs compared with ORC1 cells. ARS1211, also defined as an orc1bahs-sensitive ARS in terms of ORC binding, did not show a significant increase in plasmid loss rate. The reasons for this are unclear, but these data suggest that a reduction in ORC occupancy caused by the orc1bahs mutation does not necessarily translate into 
reduced origin firing for every ARS. In contrast to most of the sensitive ARSs, however, the three orc1bahs-resistant ARSs (ARS804, ARS716, and ARS1308) were lost at similar rates regardless of whether they were replicating in orc1bahs mutant or ORC1 cells. In addition, and consistent with the genetic data presented in Figure 4B, the sir1 $\Delta$ mutation did not substantially reduce the loss rate for any plasmid, including the orc1bahs-sensitive ARS-containing plasmids that were lost at a higher rate in orc1bahs mutant cells. Thus, the requirement of the Orc1BAH domain for ORC binding at orc1bahs-sensitive origins correlated with reduced origin function as measured by plasmid stability, indicating that a relatively local feature of the chromatin and/or DNA sequences comprising these origins was relevant to their reliance on the Orc1BAH domain for full activity.

Orclbah $\Delta$-sensitive ARSs required the $N$-terminal protein acetyltransferase gene for normal activity

NAT1 encodes the catalytic subunit of the N-terminal acetyl transferase NatA that acetylates the N-terminal residue on hundreds of yeast proteins (Polevoda and Sherman 2003). Both Orcl and the sequence-similar Sir3 protein that functions as a component of silent chromatin are $\mathrm{N}$-terminally acetylated in a NAT1-dependent manner (Geissenhoner et al. 2004; Wang et al. 2004) and this modification is required for the Sir3BAH and Orc1BAH domains to bind chromatin (Onishi et al. 2007).
Therefore, the effect of a nat $1 \Delta$ on plasmid loss rates was determined for these plasmids (Fig. 5B). The nat1s mutation elevated the loss rate for plasmids with orc1bahssensitive ARSs, but had little effect on plasmids that contained orc1bah $\Delta$-resistant ARSs. In addition, cells harboring both the nat $1 \Delta$ and orc1bahs mutations lost the orc $1 \mathrm{bah} \Delta$-sensitive ARS-containing plasmids at rates similar to cells harboring either mutation alone. These data were consistent with the NAT1-dependent N-terminal modification of Orc1 contributing to the function of the Orc1BAH domain in DNA replication, and the observation that the $\mathrm{A} 2 \mathrm{P}$ substitution in Orcl reduced ORC association with the orc1bahs-sensitive ARS1323 (Fig. 3B).

\section{Analysis of ORC-binding sites of orclbahs-sensitive and orclbahs-resistant origins}

Since the orc1bahs-sensitive origins depended heavily on the Orc1BAH domain for efficient association with ORC in vivo, a reasonable postulate was that their ORCbinding sites might deviate from the consensus ORCbinding site. Therefore, the frequency of each nucleotide within and immediately surrounding the core ORCbinding site was determined for all likely and confirmed ARSs (OriDB) in our ChIP-on-chip data set in the following three categories: the most orc1bah $\Delta$-sensitive ARSs $(n=35)$, the most orc1bah $\Delta$-resistant ARSs $(n=29)$, and all 296 ARSs (Fig. 6A). The core ORC-binding sites used
A

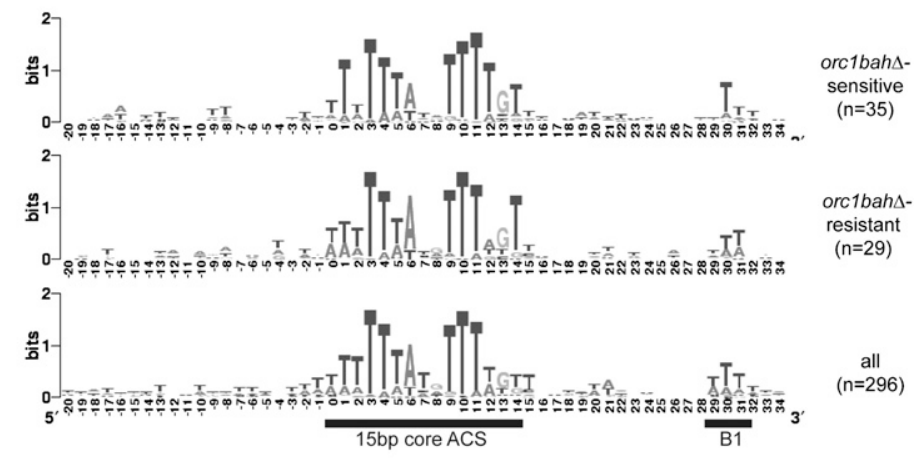

B

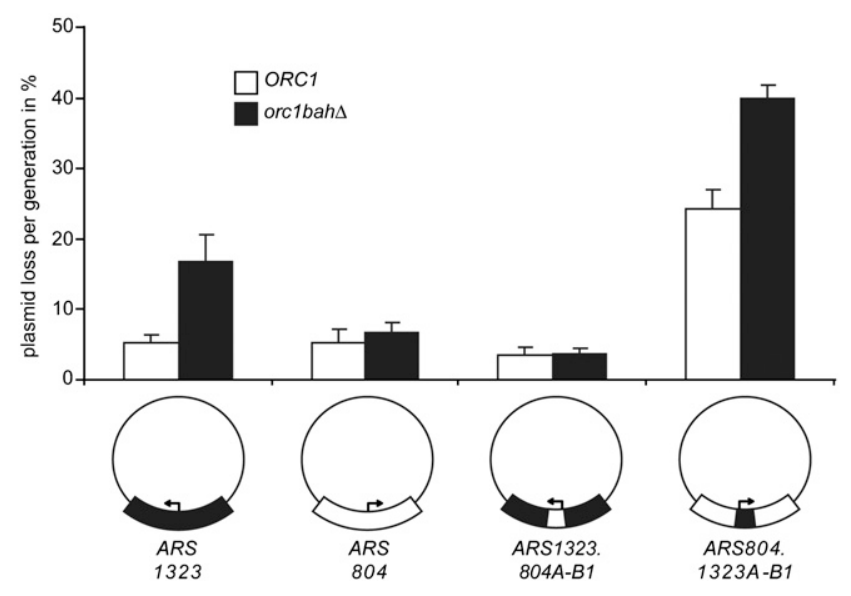

Figure 6. Difference between the core ORC-binding site of orc1bahs-sensitive and orc1bahs-resistant origins. (A) The consensus sites for the predicted ORC site and surrounding sequences were determined for the $35 \operatorname{orc} 1 \mathrm{bah} \Delta$-sensitive, the 29 orc1bahs-resistant, and all 296 of the confirmed ARSs (as listed in OriDB) using WebLogo (Crooks et al. 2004). The 15-bp core ACS (A-element) and the 3-nucleotide B1-element are indicated with a line below the consensus site shown for all 296 ARSs (Xu et al. 2006; Chang et al. 2008). (B) Plasmid loss rate data from ARS1323 (pCF1903), ARS804 (pCF1911) (as shown in Fig. 5B), and the two different ARS-ORC-binding site swaps (ARS1323.804A-B1 [pCF1982], and ARS804.1323A-B1 [pCF1981]). 
for these analyses corresponded to the predicted or verified site listed on OriDB.

The consensus ORC-binding site differed slightly between the orc1bahs-sensitive and orc1bahs-resistant ARSs in a number of positions, although overall both classes of ARSs produced consensus ORC sites that were similar to each other and to the consensus determined for all 296 ARSs. One notable difference between the consensus sites for orc1bahs-sensitive and orc1bahsresistant ARSs was the strong preference for a $\mathrm{T}$ nucleotide at position 12 in the former. The B1-element also showed some differences between orc 1 bahs $\Delta$-sensitive and orc1bahs-resistant ARSs. However, it was clear that the orc1bahs-sensitive ARSs did not exhibit a general drift away from the core consensus.

To test whether orc1bahs-sensitive and orc1bahsresistant ARSs might contain functionally different core ORC-binding sites, the predicted A-B1 bipartite ORC sites of ARS1323, an orc1bah $\Delta$-sensitive ARS, and ARS804, an orc1bahs-resistant ARS, were swapped in the context of the plasmids used in Figure 5B. If the core ORC-binding sites between these two different ARSs were functionally equivalent and unrelated to the differences documented in Figure 5B, then each fusion ARS - ARS1323 with ARS804's core ORC-binding site (ARS1323.804A-B1), and ARS804 with ARS1323's core ORC-binding site (ARS804.1323AB1) - should behave identically to the original ARS1323containing and ARS804-containing plasmids (shown again for comparison in Fig. 6B). However, we found that the two different A-B1-elements were not completely interchangeable. In a plasmid loss experiment, the ARS1323.804A-B1 fusion plasmid was lost at a twofold reduced rate compared with either original $A R S$, indicating that it was replicated more efficiently than either original plasmid. In addition, the orc1bahs mutation had no effect on the loss rate of ARS1323.804A-B1 plasmid, and, even in orc1bahs mutant cells, this plasmid was more stable than either original plasmid in a wild-type ORC1 cell. Thus, the ORC-binding site of ARS804, an orc1bahs-resistant ARS, was sufficient to suppress the orc1bahs sensitivity of ARS1323. In contrast, the ARS804.1323-A-B1 plasmid was lost at a high rate even in wild-type ORC1 cells compared with either original plasmid. Thus, ARS1323, an orc1bahs-sensitive ARS, contained elements outside of the core ORC-binding site that allowed it to work efficiently, and these elements were missing from ARS804. Taken together, these experiments made it clear that the core ORC-binding sites from orc1bahs-resistant ARS804 and orc1bahs-sensitive ARS1323 were not functionally interchangeable, and contributed to functional differences between these two ARSs.

Nucleosome positioning around the core ORC-binding site differed between orclbahs-sensitive

and $\operatorname{orc} 1 \mathrm{bah} \Delta$-resistant ARSs

The data presented in Figure 6 provided evidence that the core ORC-binding site of ARS1323 worked well in the context of this orc1bahs-sensitive ARS, but was inadequate to support robust replication in the context of orc1bahs-resistant ARS804. Given the biochemical evidence that BAH domains can bind chromatin (Onishi et al. 2007), a reasonable postulate was that something about the surrounding chromatin of ARS1323-and, presumably, other orc1bahs-sensitive ARSs-could promote ORC binding. Recent high-resolution, whole-genome nucleosome mapping experiments provide evidence that $A R S s$ exist within nucleosome-free regions (NFRs) (Lee et al. 2007; Mavrich et al. 2008), presumably to allow for ORC binding and MCM loading (Eaton et al. 2010). Therefore, high-resolution yeast genomic nucleosome maps were used to compare the sizes of NFRs between orc1baht-sensitive and orc1baht-resistant ARSs. For each ARS identified in our ORC ChIP-on-chip and predicted to exist in an NFR, the distance between the edges of the two nearest flanking nucleosomes was determined in base pairs ( $Y$-axis) and presented as box-and-whisker plots for orc1bahs-sensitive (S), orc1bahs-resistant (R), or all ARSs (X-axis, Fig. 7A). The mean NFR size in base pairs for each category of ARS is listed below the graph (Fig. 7A). The statistical significance of the mean differences in NFR sizes is shown with a $P$-value indicated in the graph (Fig. 7A). Two different published data sets were used independently in these analyses, one from Lee et al. (2007), and one from Mavrich et al. (2008), as indicated (Fig. 7A). These analyses provided evidence that NFRs containing orc1bahs-sensitive ARSs were smaller in size compared with the NFRs containing orc1bahs-resistant ARSs.

To gain further insights into the nucleosome positioning differences surrounding these different classes of origins, the nucleosome organization upstream of and downstream from the A-element (ACS) was examined (Fig. 7B). A graph was generated in which the average tiling array nucleosome signal ( $Y$-axis) (Lee et al. 2007) was plotted as a function of position relative to the ACS ( $X$-axis) (Fig. 7B). All of the ACS matches were aligned by their T-rich strand, with position 0 referring to the first nucleotide on the T-rich strand of the core ACS as indicated in Figure 6A. Ordered and phased nucleosomes were detected both upstream of $\left(5^{\prime}\right.$ of the ACS as indicated in Fig. 6A; such nucleosomes were given a minus designation [i.e., -1 , -2 , and so on]) and downstream from the ACS for each class of origins (Eaton et al. 2010). However, nucleosome positioning around orc1bahs-sensitive (S) ARSs was unique. Specifically, both the -2 and -1 nucleosomes immediately upstream of the ACS were shifted closer to the ACS. These data provided evidence that the smaller NFR observed in orc1bahs-sensitive origins (Fig. 7A) was associated with a change in nucleosome organization upstream of these origins. In particular, it is notable that the linker region between the -3 and -2 nucleosome is larger for orc1bah $\Delta$-sensitive origins, raising the possibility that there is some feature of this region that is displacing nucleosomes toward the ARS (Fig. 7B). Importantly, the same analysis performed with recently obtained ChIP-seq nucleosome positioning data (Eaton et al. 2010) supported this idea, and a heat map analysis revealed that the enhanced linker space between the -3 and -2 nucleosomes did indeed occur at many ARSs within the orc1bah $\Delta$-sensitive class, and was not simply due to one 
A

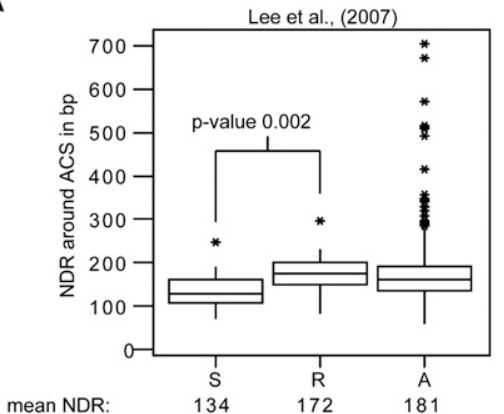

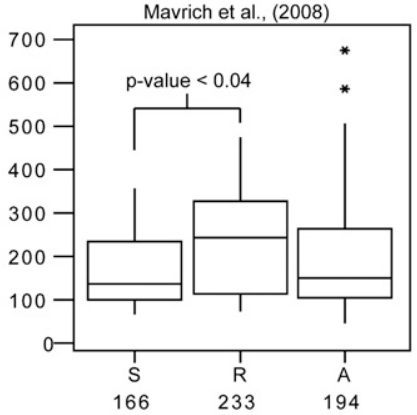

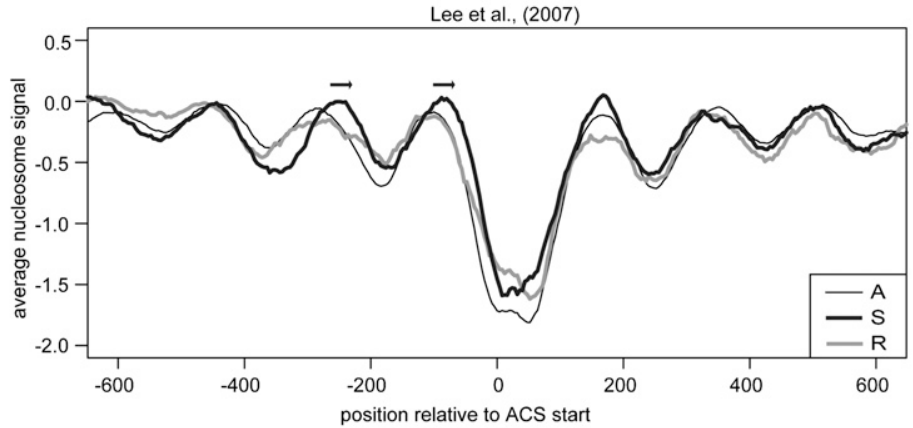

Figure 7. Differences in nucleosome positioning surrounding orc $1 b a h \Delta$-sensitive and orc1bah $\Delta$-resistant origins. (A) The average size in base pairs of the nucleosome-depleted regions (NDRs; also called NFRs) containing the orc1bah $\Delta$-sensitive (S), orc1bahs-resistant $(\mathrm{R})$, and all ARSs identified in our ChIP-on-chip and predicted to exist within an NFR was determined. The data are presented as a box-and-whiskers plot ( $Y$-axis indicates size of NFR in base pairs) and the $P$-value confidence value for comparisons of the means of the $S$ and R NFRs are indicated. The mean NFR for each class of ARS is indicated below the $X$-axis. The asterisk $\left({ }^{*}\right)$ indicates outlier ARSs in each category. Two different data sets were used independently in these analyses, from Lee et al. (2007) and Mavrich et al. (2008). (B) The average nucleosome signal ( $Y$-axis) was plotted for each nucleotide position relative to the T-rich strand of the ACS (with the 0 position of the ACS as indicated in Fig. 6A) for $\operatorname{orc} 1 b a h \Delta$-sensitive (S), orc1bah $\Delta$-resistant (R), and all other (A) ARSs. The nucleosome positioning data set was from Lee et al. (2007). Shifts in the -1 and -2 nucleosomes and the linker space between the -3 and -2 nucleosomes for the $\mathrm{S}$ class are indicated with arrows. This analysis was repeated with a more recently generated whole-genome nucleosome positioning data set with similar results (Supplemental Fig. S4).

C

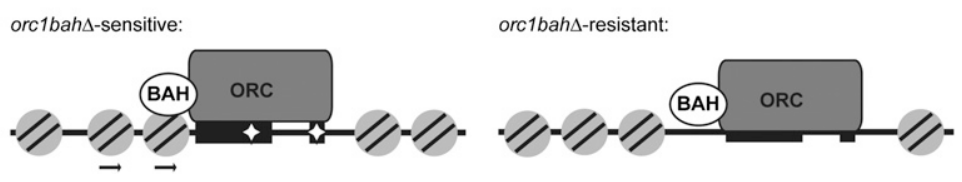

or two ARSs in the group having unusually large gaps between their -3 and -2 nucleosomes (Supplemental Fig. S3). Thus, orc1bah $\Delta$-sensitive origins exhibited a unique local chromatin structure characterized by altered nucleosome positioning.

\section{Discussion}

The previously established role for the Saccharomyces cerevisiae Orc1BAH domain is to function in the process of transcriptional silencing via a direct physical interaction with the nonessential silencing protein Sirl (Fox and McConnell 2005). Yeast cells lacking the Orc1BAH domain show no viability defects, and orc1bahs and sir1s mutations produce the same transcriptional silencing phenotypes alone and in combination, supporting the notion that the Orc1BAH domain of yeast functions in transcriptional silencing but not the essential process of DNA replication. This study re-examined a possible role for the Orc1BAH domain in DNA replication by comparing ORC binding in wild-type and orc1bahs mutant cells genome-wide. This comparison provided evidence that the Orc1BAH domain contributed to ORC's association with the majority of DNA replication origins. In addition, for a class of orc1bahs-sensitive origins, the Orc1BAH domain was important for normal origin activity in both chromosomal and plasmid contexts.
The Orc1BAH domain contributed differentially to ORC's association with individual yeast origins, such that origin dependence on the Orc1BAH domain for ORC binding varied along a continuum from highly dependent (orc1bahs-sensitive) to independent (orc1bahs-resistant) origins. This variation helps reconcile a role for the Orc1BAH domain in DNA replication, and the observation that the Orc1BAH domain is dispensable for viability (Bell et al. 1995): orc1bahs mutant cells are viable because, in these cells, most replication origins remain partially or fully functional. Indeed, orc $1 b a h \Delta$-resistant origins and ARS1 fired as efficiently in orc1bahs mutant cells as they did in wild-type cells. However, orc1bahs-sensitive origins exhibited reduced activity in orc1bahs mutant cells compared with wild-type cells. Analyses of orc1bahssensitive origins uncovered features in their ORC-binding sites and surrounding nucleosomes that provided a reasonable hypothesis to explain their Orc1BAH dependence.

\section{An accessory element that contributed to yeast origin recognition by $\mathrm{ORC}$}

An ORC-binding site is necessary but not sufficient for the function of yeast origins. The accessory DNA elements that enhance origin activity studied to date function at a step after origin recognition by ORC. For example, the B2-element of ARS1 contributes to pre-RC assembly but 
not ORC binding at ARS1 (Wilmes and Bell 2002). Similarly, the Abf1-binding site (B3-element) is required for the full activity of ARS1 (Marahrens and Stillman 1992), but Abf1 does not enhance ORC binding to ARS1 in vitro (Rao and Stillman 1995). In contrast, this study provided evidence that some origins possess "accessory elements" that help with the ORC-binding step. In particular, the orc1bahs-sensitive origins in this study, by definition, required the Orc1BAH domain, a nucleosome-binding module (Onishi et al. 2007), to bind ORC efficiently in vivo. These orc1bah $\Delta$-sensitive origins possessed a unique ORCbinding site consensus sequence and an associated distinctive nucleosome structure. Precisely substituting the ARS804 (orc1bahs-resistant) ORC-binding site with that from ARS1323 (orc1bahs 4 -sensitive) created a fusion ARS that functioned poorly compared with either parent ARS, even in wild-type ORC1 cells. Combined with recent biochemical data indicating that the BAH domains are nucleosome-binding modules, the data presented in this report support a model in which ORC recognizes a composite binding site within orc1bahs-sensitive origins that consists of both a nucleosome and an A-B1 bipartite ORCbinding site (Fig. 7C).

\section{Positioned nucleosomes and origin function}

Nucleosome positioning has been implicated in regulating origin activity in yeast, but, in most examples, positioned nucleosomes inhibit rather than enhance origin activity. For example, negative regulation of replication origins by Sir2 requires an element in the vicinity of these origins that binds a positioned nucleosome (Casey et al. 2008; Crampton et al. 2008). Thus, at Sir2-regulated origins, a positioned nucleosome normally acts as an inhibitor of origin function. Focused studies of the ARS1 origin have also provided evidence that positioned nucleosomes can inhibit origin activity, particularly when nucleosomes are forced into functional elements of ARS1, such as the ORCbinding site itself (Simpson 1990; Lipford and Bell 2001). When ORC binds to its site in ARS1, it helps position neighboring nucleosomes, suggesting that there may be competition between ORC and nucleosome binding at ARSs, and nucleosomes positioned at ARS1 by ORC can enhance MCM loading without affecting ORC binding (Lipford and Bell 2001). In this study, orc1bahs-sensitive origins were found to exist within a distinct nucleosome configuration: Their NFRs were, on average, smaller than the NFRs that contained other yeast origins. Furthermore, the smaller NFR was due at least in part to a nucleosome on the $5^{\prime}$ end of these origins encroaching into the ORCbinding site. Significantly, structural studies of ARS1 in vitro (Lee and Bell 1997; Chastain et al. 2004; Chen et al. 2008) indicate that the Orcl subunit should be positioned nearest the A-element of the ORC-binding site that abuts this -1 nucleosome. It is also notable that both the -2 and -1 nucleosomes are displaced toward the A-element of the ORC-binding site of orc1bahs $\Delta$-sensitive origins, and that there is a larger-than-average nucleosome-free linker between the -3 and -2 nucleosomes. Thus, we propose that, at orc1bahs $\Delta$-sensitive origins, the nucleosome positioned $5^{\prime}$ of the NFR directly or indirectly interacts with the
Orc1BAH domain and promotes ORC-origin association (Fig. 7C). Since the nucleosomes immediately flanking ARSs (i.e., -1 and +1 ) in vivo are positioned in part by ORC itself (Eaton et al. 2010), the precise nucleosomal architecture at orc1bahs-sensitive ARSs may result from a more complex interplay between ORC, nucleosomes, and possibly other factors such as local DNA sequence and/or nonhistone proteins, than depicted in this simplified cartoon.

A number of studies have implicated chromatinmodifying enzymes in the control of origin activity in yeast. For example, the Rpd3 histone deacetylase modulates the timing of origin firing for a large number of origins (Vogelauer et al. 2002; Aparicio et al. 2004; Knott et al. 2009). Moreover, direct acetylation of nucleosomes by a tethered histone acetyltransferase can advance the firing time of a local replication origin, suggesting that acetylated nucleosomes enhance some aspect of origin firing (Vogelauer et al. 2002). And, as mentioned above, Sir2, a histone deacetylase, negatively regulates specific origins through an element that binds a positioned nucleosome (Crampton et al. 2008). The most direct model to explain this effect posits that acetylation of this nucleosome enhances the activity of these Sir2-regulated origins, presumably by enhancing MCM loading during pre-RC assembly. Therefore, it seemed probable that the positioned nucleosomes that enhance ORC binding at orc1bahs-sensitive origins might require a specific modification or chromatin remodeling activity for their positioning and/or ability to interact with the Orc1BAH domain. Several genes encoding chromatin modifiers implicated previously in origin control or shown to interact genetically with specific $O R C$ alleles were tested for their roles in controlling activity of orc1bahs-sensitive origins (Supplemental Fig. S4; Suter et al. 2004; Irlbacher et al. 2005; Lynch et al. 2005). With the exception of the NAT1 gene required for acetylation of the Orc1BAH, no mutation tested came close to producing a phenocopy of the orc1bahs mutation (i.e., specifically reduced stability of orc1bahs-sensitive ARS-containing plasmids without affecting orc $1 b a h \Delta$-resistant ARS-containing plasmids), including hat $1 \Delta$. The hat $1 \Delta$ mutation was important to test because the Hat 1 protein, similar to the core histones, was underrepresented in the IP from orc1bahs cells compared with ORC1 cells (Supplemental Table S1). However, many additional modifiers are worth consideration, and it is possible that only a specific allele of a particular gene might be relevant. Functional identification of the elements near orc1bah $\Delta$-sensitive origins that establish both their local nucleosome structure and reliance on the Orc1BAH domain is ongoing, and will be necessary for understanding the mechanisms controlling orc1bahs -sensitive origin structure and function.

\section{The metazoan connection}

Accessory elements that contribute to ORC binding to yeast origins, the first step in the origin cycle, have likely not been a major focus of replication studies for the simple reason that yeast ORC clearly binds to a specific DNA sequence that can be identified in all yeast origins. 
However, in metazoans, ORC does not show this level of DNA sequence specificity, and thus non-DNA sequence factors, such as specific chromatin structures, are likely an important component of origin recognition by ORC. Therefore, in terms of ORC's origin recognition mechanisms, orc 1 bah $\Delta$-sensitive origins may turn out to work more similarly to metazoan origins, especially since, by definition, they rely heavily on the conserved Orc1BAH domain for ORC binding. It will be interesting to learn how this type of binding, as opposed to binding that relies more heavily on direct ORC-DNA interactions (as is likely true for orc1bahs-resistant origins), influences ORC's other activities that are important for pre-RC assembly and, ultimately, for origin firing. Significantly, the 2D origin mapping data provided evidence that the Orc1BAH domain, or ORC occupancy as affected by the Orc1BAH domain, can affect dynamics of origin firing qualitatively, increasing the heterogeneity in sizes of origin bubble intermediates. These data raise the possibility that origin selection mechanisms and the ultimate nature of origin firing during $\mathrm{S}$ phase (i.e., timing and/or efficiency of firing) may be connected more intimately in budding yeast than thought previously, and thus more similar to origin control in Schizosaccharomyces pombe (Wu and Nurse 2009) and, by extension, metazoans than has been appreciated. The data presented here and elsewhere (Noguchi et al. 2006; Onishi et al. 2007) provide evidence that Orc1BAH-chromatin interactions are a conserved feature of ORC's selection of replication origins from yeast to humans. Thus, a class of yeast orc1bahssensitive origins can provide a genetically tractable experimental system to examine evolutionarily conserved mechanisms of origin selection and firing, the relationship between these two events, and their dependence on local chromatin structure.

\section{Materials and methods}

\section{Yeast genetics and molecular biology}

Yeast strains were constructed using standard methods (Guthrie and Fink 1991), and were all of the W303 background (Supplemental Table S4). Plasmids for plasmid loss rate experiments were constructed using pARS1/WTA (Marahrens and Stillman 1992). The ARS1 fragment was excised by HindIII and EcoRI digestion, and a linker containing BamHI and NotI restriction sites was ligated in its place to generate pARS1/PM (pCF1897). Approximately $1-\mathrm{kb}$ chromosomal regions containing ARSs of interest were PCR-amplified with primers that contained BamHI and NotI restriction sites, and were inserted into the BamHI/ NotI sites within the linker of pCF1897 (see Supplemental Table S5 for oligo sequences). Inserts were sequenced prior to plasmid loss rate experiments. For the A-B1-element swap experiments (Fig. 6B), the essential A-element (ACS) for ARS1323 and ARS804 was verified by generating two individual Sall-marked deletions of nucleotides $1-7$ and $9-15$ of predicted ACSs (nimACS2 for ARS1323, and nimACS1 for ARS804) (Xu et al. 2006). The plasmids containing the ACS mutations failed to transform yeast, indicating that the predicted A-element was part of a single essential ORC-binding site (P Müller, unpubl.; data not shown). Plasmids containing the A-B1-element swaps, pARS1323.804A-B1 and pARS804.1323A-B1, were engi- neered using the two-step recombination method described in Nieduszynski et al. (2005). The two A-B1 sequences (33 nucleotides total) were swapped precisely, as shown in the following sequences. The sequences correspond to the -1 to -31 nucleotides in the WebLogo alignments. Fifteen nucleotides of the receptor ARS sequence are shown on either side of the A-B1-element from the donor ARS. Sequences are shown $5^{\prime}$ to $3^{\prime}$, and were engineered to maintain orientation of $\mathrm{A}-\mathrm{B} 1$ in the parent ARS.

$$
\begin{aligned}
& \text { pARS1323.804A-B1: } \\
& \text { ACS B1 } \\
& \text { atggtttatttatgaAAAGTTAATATTTAGTACCGATACTATACATTAcaataagtgcttatt } \\
& \text { pARS804.1323A-B1: } \\
& \text { B1 ACS } \\
& \text { cgtttgtttataaaaATTTTGAGTATAAAGAACATAAACATAAACAATtccgaaaaatcaaaa }
\end{aligned}
$$

Supplemental Table S4 shows sequences relevant to cloning all plasmids used in plasmid loss experiments.

\section{Immunopurification of ORC and associated nuclear proteins followed by iTRAQ analyses}

Yeast nuclei from $12 \mathrm{~L}$ of $O R C 1$ and orc 1 bahs cells grown to an $\mathrm{A}_{600}$ of 1 were prepared by spheroplasting. DNA content was determined by measuring $A_{260}$ of lysed nuclei with an $A_{260}$ of $1=$ $50 \mu \mathrm{g} / \mathrm{mL}$ DNA. Average yield per liter of yeast culture was $\sim 4000 \mu \mathrm{g}$ of DNA. Nuclei were lysed in NP-40 lysis buffer (150 $\mathrm{mM} \mathrm{NaCl}, \mathrm{K}_{2} \mathrm{HPO}_{4}$ at $\mathrm{pH} 7.2,1 \%$ NP-40), and chromatin was fragmented by MNase treatment (30 U of MNase/100 $\mu \mathrm{g}$ of DNA for $45 \mathrm{~min}$ at $4^{\circ} \mathrm{C}$ ) to generate mono-, di-, and trinucleosomalsized fragments (Supplemental Fig. S1). ORC was immunoprecipitated with a cocktail of two mouse monoclonal antibodies raised against Orc1 (4H3 and 3E9).

The solution of immunoprecipitated proteins was precipitated with TCA and subjected to relative quantitation using the iTRAQ reagent kit (Applied Biosystems). Precipitates estimated at between $10 \mu \mathrm{g}$ and $50 \mu \mathrm{g}$ for each (matched between wild-type and mutant IPs) were then solubilized, reduced, alkylated, and proteolyzed with trypsin. The trypsinized peptides were labeled with iTRAQ reagents 114 and 117, respectively, according to the manufacturer's instructions, and mixed. Labeled peptides were purified by strong cation exchange, desalted by $\mathrm{C}_{18}$ solid-phase extraction, and concentrated by vacuum centrifugation. The mixed, labeled digests were then separated for LC/MALDI as described (Huttlin et al. 2009), except that solvent B consisted of $65 \%(\mathrm{v} / \mathrm{v})$ acetonitrile, $5 \%(\mathrm{v} / \mathrm{v})$ isopropyl alcohol, and $0.1 \%(\mathrm{v} / \mathrm{v})$ trifluoroacetic acid (TFA) in water. The MALDI matrix used for spot deposition during LC separation contained $4 \mathrm{mg} / \mathrm{mL} \alpha$-cyano-4-hydroxycinnamic acid, $75 \%(\mathrm{v} / \mathrm{v})$ acetonitrile, $0.1 \%(\mathrm{v} / \mathrm{v})$ TFA, $30 \mathrm{fmol} / \mu \mathrm{L}$ adrenocorticotropic hormone fragment 18-39 (as internal standard), and $10 \mathrm{mM}$ ammonium phosphate in water. The matrix was mixed with HPLC eluent in a static mixing tee at a ratio of $4: 1$ (matrix:eluent) at a combined flow rate of $1 \mu \mathrm{L} / \mathrm{min}$. Spots were deposited in 0.2 -min increments between 40 and $250 \mathrm{~min}$, for a total of 1050 spots, onto a stainless steel MALDI target (Applied Biosystems Opti-TOF). Two LC/ MALDI separations of the digested, labeled, mixed peptides were performed. Additional details on MALDI mass spectrometry and post-acquisition data processing and analyses are described in the legend for Supplemental Table S1.

\section{Determination of ORC's association with chromosomes}

Exponentially growing yeast cultures were harvested, and samples were processed into a chromatin-containing pellet fraction (pellet) and a supernatant fraction containing soluble proteins (soluble) after Nonidet P-40 lysis of yeast spheroplasts as described (Liang and Stillman 1997). Equivalent amounts of these 
fractions were loaded onto SDS-polyacrylamide gels, transferred to nitrocellulose, and probed with antibodies against Orc1, Orc2, Orc3, and Orc4, as well as Fkh1 and Sis $1 . \mathrm{A}_{600}$ cell equivalents of $0.375(1 \times)$ and $0.75(2 \times)$ were analyzed per lane in Figure $1 \mathrm{C}$. The $\alpha$-Orcl antibodies (Bose et al. 2004) and $\alpha$-Fkhl antibodies (Casey et al. 2008) have been described. Antibodies against Sis1 were a gift from Elizabeth Craig (Yan and Craig 1999).

\section{Analysis of ORC binding in ORC1 and orc1bahs cells}

Cultures were grown to an $\mathrm{A}_{600}$ of 1.0, and ChIPs were performed as described (Casey et al. 2008) using a monoclonal antibody against Orcl protein (Bose et al. 2004). The chromatin was sheared by sonication to an average size of $0.6 \mathrm{~kb}$. IP and total DNAs were purified using a QIAquick PCR purification kit (Qiagen).

For ORC ChIP-on-chip, IPs and total DNA were amplified using ligation-mediated PCR and mailed to Nimblegen for hybridization to high-density tiled arrays (2006-10-12_Ansari_tiling_51mer). For each ChIP-on-chip experiment, the ChIP sample was labeled with $\mathrm{Cy} 3$ and the total (sheared genomic DNA) was labeled with Cy5. The $\log 2$ ratios of Cy3 to Cy5 intensities for each probe on the array were determined and provided by Nimblegen. IPs over total were obtained from the values extracted from the $S$. cerevisiae tiling microarray for each feature. The most repetitive probes $(1.1 \%)$ were removed from the data set. Peaks were identified by ChiPOTle (Buck et al. 2005) with a $P$-value cutoff of $10^{-20}$, assuming a Gaussian distribution and using a window size of $400 \mathrm{bp}$ and step size of $75 \mathrm{bp}$. From the resulting list of peaks, peaks with a $P$-value $<10^{-30}$ were included in further analysis. This $P$-value cutoff was judged to give the best compromise between retaining as many confirmed ORC peaks as possible, while removing smaller peaks that would be difficult to follow-up experimentally. Peak area was calculated as the sum of the $\log 2$ ratios of all probes that were contained within the peak.

For ARS-specific ChIPs, real-time qPCR was used and yeast cultures were grown to an $\mathrm{A}_{600}$ of $\sim 0.4$ and then arrested in G1 with $3 \mu \mathrm{M} \alpha$-factor (final arrested culture: $\mathrm{A}_{600} \sim 1.0$ ). ORCdirected ChIPs were performed with an anti-Orcl monoclonal antibody (Bose et al. 2004), and MCM-directed ChIPs were performed with an anti-Mcm2 polyclonal antibody (Santa Cruz Biotechnology) or an anti-Mcm2 monoclonal antibody (generated in the laboratory of Bruce Stillman) and protein G Dynabeads (Invitrogen). qPCR reactions containing the SYBR Green Mix (Bio$\mathrm{Rad}$ ) and gene-specific primers (Fig. 3A; Supplemental Table S4) were performed. Reaction products were analyzed by iQ optical system software (Bio-Rad). All reactions were performed in duplicate with at least two biological replicates per experiment.

\section{Determination of plasmid loss rates}

Plasmid loss rate assays and calculations were performed as described (Pappas et al. 2004). At least five independent cultures for each plasmid were inoculated from single colonies and grown overnight at $30^{\circ} \mathrm{C}$ in CAS (casamino acid media, lacking uracil) media for $\sim 18 \mathrm{~h}$. The cultures were diluted to an $\mathrm{OD}_{600}$ of $\sim 0.001$ into CAS supplemented with uracil and grown at $30^{\circ} \mathrm{C}$ to an $\mathrm{OD}_{600}$ of $\sim 1$. Dilutions of both cultures were plated onto CAS + ALL and CAS plates at the start of the experiment and at the end, and colonies were counted $\sim 48 \mathrm{~h}$ later. Generational loss rates were calculated as described (Donato et al. 2006). Briefly, colony counts were used to calculate the initial (\%I) and final $(\% \mathrm{~F})$ percentages of cells containing plasmid. The loss rate $(L)$ was calculated according to the formula $L=1-(\% \mathrm{~F} / \% \mathrm{I})(1 / n)$, where $n$ equals the number of generations (cell doublings).

\section{Bioinformatic analyses of ChIP-on-chip data}

ACS sequences and coordinates for ORC peaks corresponding to "likely" and "confirmed" ARSs (as annotated in OriDB) were retrieved from OriDB (http://www.oridb.org) and Saccharomyces Genome Databae (http://www.yeastgenome.org). Sequence logos of the 15-bp ACS \pm 20 bp were generated for orc1bahssensitive (ratio $<0.33, n=35$ ), orc 1 bah $\Delta$-resistant (ratio $>0.8, n=$ $29)$, and all peaks in our data set $(n=296)$ using WebLogo (Fig. 6A; Crooks et al. 2004).

To determine the size of the NFRs (i.e., also referred to as nucleosome-depleted regions [NDRs]) surrounding various groups of ARSs (Fig. 7A) a Perl script was written specifically for this purpose. NFRs were defined by the $3^{\prime}$ end of the upstream and the 5 ' end of the downstream nucleosome sequences relative to the ACS on the T-rich strand. For Figure 7B, the 4-bp resolution tiling array nucleosome mapping data from Lee et al. (2007) were used. Briefly, probes in the area local to each A-element were assigned a position relative to the location of the T-rich copy of the ACS motif. Defining a bin every $4 \mathrm{bp}$ then aligned the probes, and their average distance to the ACS labeled the bins. The intensity scores for all probes were then averaged per bin to obtain the graph (Fig. 7B). The analysis was repeated with a more recently generated whole-genome nucleosome positioning data set (Eaton et al. 2010), and the same results were obtained (Supplemental Fig. S4)

\section{Acknowledgments}

We are grateful to Timothy Hoggard (Fox laboratory) for help establishing the plasmid loss rate assay during his rotation, and Ulrika Müller (Fox laboratory) for help with 2D origin mapping. We thank Dr. Elizabeth Craig (Department of Biochemistry, University of Wisconsin-Madison) for antibodies against Sis1, Dr. Paul Kaufman (Program in Gene Function and Expression, University of Massachusetts Medical School, Worcestire) for yeast strains, Dr. Greg Barrett-Wilt of the Mass Spectrometry Facility (Biotechnology Center, University of Wisconsin-Madison) for performing the iTRAQ analyses and guidance in data interpretation, and Drs. Bruce Stillman (Cold Spring Harbor Laboratory) and Oscar Aparicio (Molecular and Computational Biology Program, University of Southern California) for providing antibodies and/or strains for MCM ChIP experiments. We also extend special thanks to Dr. Audrey Gasch (Laboratory of Genetics, University of Wisconsin-Madison) for allowing her student (D.J.H.) to help us with data analyses, and Dr. Patricia J. Kiley (Department of Biomolecular Chemistry, University of Wisconsin-Madison) for discussing ideas throughout the course of this study, and helpful comments on the manuscript. Finally, we are grateful to JinWoo Lee (Laboratory of Dr. Colin Jefcoate, Program in Endrocrinology and Reproductive Physiology, University of Wisconsin-Madison) for help with qPCR. A grant from the National Institutes of Health (GM56890 to C.A.F.) and a Vilas Associate Award from the University of Wisconsin-Madison graduate school (to C.A.F.) supported the majority of this work. An ARRA supplement to GM56890 supported S.P. A Leukemia and Lymphoma Society Fellowship (no. 5255-07) supported E.S. A position on the Molecular Bioscience Training Grant T32 GM007215 from the National Institute of General Medical Sciences supported D.J.H. A grant from the NIH (HG004279) and a Whitehead Scholar Award supported D.M.M.

\section{References}

Aladjem MI. 2007. Replication in context: Dynamic regulation of DNA replication patterns in metazoans. Nat Rev Genet 8: 588-600. 
Aparicio JG, Viggiani CJ, Gibson DG, Aparicio OM. 2004. The Rpd3-Sin3 histone deacetylase regulates replication timing and enables intra-S origin control in Saccharomyces cerevisiae. Mol Cell Biol 24: 4769-4780.

Bell SP. 2002. The origin recognition complex: From simple origins to complex functions. Genes Dev 16: 659-672.

Bell SP, Dutta A. 2002. DNA replication in eukaryotic cells. Annu Rev Biochem 71: 333-374.

Bell SP, Kobayashi R, Stillman B. 1993. Yeast origin recognition complex functions in transcription silencing and DNA replication. Science 262: 1844-1849.

Bell SP, Mitchell J, Leber J, Kobayashi R, Stillman B. 1995. The multidomain structure of Orclp reveals similarity to regulators of DNA replication and transcriptional silencing. Cell 83: 563-568.

Bose ME, McConnell KH, Gardner-Aukema KA, Muller U, Weinreich M, Keck JL, Fox CA. 2004. The origin recognition complex and Sir4 protein recruit Sirlp to yeast silent chromatin through independent interactions requiring a common Sirlp domain. Mol Cell Biol 24: 774-786.

Buchberger JR, Onishi M, Li G, Seebacher J, Rudner AD, Gygi SP, Moazed D. 2008. Sir3-nucleosome interactions in spreading of silent chromatin in Saccharomyces cerevisiae. Mol Cell Biol 28: 6903-6918.

Buck MJ, Nobel AB, Lieb JD. 2005. ChIPOTle: A user-friendly tool for the analysis of ChIP-chip data. Genome Biol 6: R97. doi: 10.1186/gb-2005-6-11-r97.

Callebaut I, Courvalin JC, Mornon JP. 1999. The BAH (bromoadjacent homology) domain: A link between DNA methylation, replication and transcriptional regulation. FEBS Lett 446: 189-193.

Casey L, Patterson EE, Muller U, Fox CA. 2008. Conversion of a replication origin to a silencer through a pathway shared by a Forkhead transcription factor and an S phase cyclin. Mol Biol Cell 19: 608-622.

Chang F, Theis JF, Miller J, Nieduszynski CA, Newlon CS, Weinreich M. 2008. Analysis of chromosome III replicators reveals an unusual structure for the ARS318 silencer origin and a conserved WTW sequence within the origin recognition complex binding site. Mol Cell Biol 28: 5071-5081.

Chastain PD II, Bowers JL, Lee DG, Bell SP, Griffith JD. 2004. Mapping subunit location on the Saccharomyces cerevisiae origin recognition complex free and bound to DNA using a novel nanoscale biopointer. I Biol Chem 279: 3635436362.

Chen Z, Speck C, Wendel P, Tang C, Stillman B, Li H. 2008. The architecture of the DNA replication origin recognition complex in Saccharomyces cerevisiae. Proc Natl Acad Sci 105: 10326-10331.

Connelly JJ, Yuan P, Hsu HC, Li Z, Xu RM, Sternglanz R. 2006. Structure and function of the Saccharomyces cerevisiae Sir3 BAH domain. Mol Cell Biol 26: 3256-3265.

Crampton A, Chang F, Pappas DL Jr, Frisch RL, Weinreich M. 2008. An ARS element inhibits DNA replication through a SIR2-dependent mechanism. Mol Cell 30: 156-166.

Crooks GE, Hon G, Chandonia JM, Brenner SE. 2004. WebLogo: A sequence logo generator. Genome Res 14: 1188-1190.

Cubizolles F, Martino F, Perrod S, Gasser SM. 2006. A homotrimer-heterotrimer switch in Sir2 structure differentiates rDNA and telomeric silencing. Mol Cell 21: 825-836.

Cvetic C, Walter JC. 2005. Eukaryotic origins of DNA replication: Could you please be more specific? Semin Cell Dev Biol 16: $343-353$.
Diffley JF. 2004. Regulation of early events in chromosome replication. Curr Biol 14: R778-R786. doi: 10.1016/j.cub.2004. 09.019 .

Donato JJ, Chung SC, Tye BK. 2006. Genome-wide hierarchy of replication origin usage in Saccharomyces cerevisiae. PLoS Genet 2: e141. doi: 10.1371/journal.pgen.0020141.

Eaton ML, Galani K, Kang S, Bell SP, MacAlpine DM. 2010. Conserved nucleosome positioning defines replication origins. Genes Dev 24: 748-753.

Fox CA, McConnell KH. 2005. Toward biochemical understanding of a transcriptionally silenced chromosomal domain in Saccharomyces cerevisiae. J Biol Chem 280: 8629-8632.

Fox CA, Loo S, Dillin A, Rine J. 1995. The origin recognition complex has essential functions in transcriptional silencing and chromosomal replication. Genes Dev 9: 911-924.

Gavin KA, Hidaka M, Stillman B. 1995. Conserved initiator proteins in eukaryotes. Science 270: 1667-1671.

Geissenhoner A, Weise C, Ehrenhofer-Murray AE. 2004. Dependence of ORC silencing function on NatA-mediated $\mathrm{N} \alpha$ acetylation in Saccharomyces cerevisiae. Mol Cell Biol 24: 10300-10312.

Gilbert DM. 2004. In search of the holy replicator. Natl Rev 5: $848-855$

Goodwin GH, Nicolas RH. 2001. The BAH domain, polybromo and the RSC chromatin remodelling complex. Gene 268: 1-7.

Guthrie C, Fink GR. 1991. Guide to yeast genetics and molecular biology. Methods in enzymology 194. Academic Press, San Diego, CA.

Hou Z, Danzer JR, Fox CA, Keck JL. 2006. Structure of the Sir3 protein bromo adjacent homology (BAH) domain from $S$. cerevisiae at 1.95 A resolution. Protein Sci 15: 1182-1186.

Huttlin EL, Chen X, Barrett-Wilt GA, Hegeman AD, Halberg RB, Harms AC, Newton MA, Dove WF, Sussman MR. 2009. Discovery and validation of colonic tumor-associated proteins via metabolic labeling and stable isotopic dilution. Proc Natl Acad Sci 106: 17235-17240.

Irlbacher H, Franke J, Manke T, Vingron M, Ehrenhofer-Murray AE. 2005. Control of replication initiation and heterochromatin formation in Saccharomyces cerevisiae by a regulator of meiotic gene expression. Genes Dev 19: 1811-1822.

Knott SR, Viggiani CJ, Tavare S, Aparicio OM. 2009. Genomewide replication profiles indicate an expansive role for Rpd3L in regulating replication initiation timing or efficiency, and reveal genomic loci of Rpd3 function in Saccharomyces cerevisiae. Genes Dev 23: 1077-1090.

Lee DG, Bell SP. 1997. Architecture of the yeast origin recognition complex bound to origins of DNA replication. Mol Cell Biol 17: 7159-7168.

Lee W, Tillo D, Bray N, Morse RH, Davis RW, Hughes TR, Nislow C. 2007. A high-resolution atlas of nucleosome occupancy in yeast. Nat Genet 39: 1235-1244.

Liang C, Stillman B. 1997. Persistent initiation of DNA replication and chromatin-bound MCM proteins during the cell cycle in cdc6 mutants. Genes Dev 11: 3375-3386.

Lipford JR, Bell SP. 2001. Nucleosomes positioned by ORC facilitate the initiation of DNA replication. Mol Cell 7: 21-30.

Lynch PJ, Fraser HB, Sevastopoulos E, Rine J, Rusche LN. 2005. Sumlp, the origin recognition complex, and the spreading of a promoter-specific repressor in Saccharomyces cerevisiae. Mol Cell Biol 25: 5920-5932.

Marahrens Y, Stillman B. 1992. A yeast chromosomal origin of DNA replication defined by multiple functional elements. Science 255: 817-823.

Mavrich TN, Ioshikhes IP, Venters BJ, Jiang C, Tomsho LP, Qi J, Schuster SC, Albert I, Pugh BF. 2008. A barrier nucleosome 
model for statistical positioning of nucleosomes throughout the yeast genome. Genome Res 18: 1073-1083.

Mechali M. 2001. DNA replication origins: From sequence specificity to epigenetics. Nat Rev Genet 2: 640-645.

Newlon CS, Theis JF. 1993. The structure and function of yeast ARS elements. Curr Opin Genet Dev 3: 752-758.

Nieduszynski CA, Blow JJ, Donaldson AD. 2005. The requirement of yeast replication origins for pre-replication complex proteins is modulated by transcription. Nucleic Acids Res 33: $2410-2420$.

Nieduszynski CA, Hiraga S, Ak P, Benham CI, Donaldson AD. 2007. OriDB: A DNA replication origin database. Nucleic Acids Res 35: D40-D46. doi: 10.1093/nar/gk1758.

Noguchi K, Vassilev A, Ghosh S, Yates JL, DePamphilis ML. 2006. The BAH domain facilitates the ability of human Orc1 protein to activate replication origins in vivo. $E M B O J \mathbf{2 5}$ : 5372-5382.

Onishi M, Liou GG, Buchberger JR, Walz T, Moazed D. 2007. Role of the conserved Sir3-BAH domain in nucleosome binding and silent chromatin assembly. Mol Cell 28: 1015-1028.

Palacios DeBeer MA, Muller U, Fox CA. 2003. Differential DNA affinity specifies roles for the origin recognition complex in budding yeast heterochromatin. Genes Dev 17: 1817-1822.

Pappas DL Jr, Frisch R, Weinreich M. 2004. The NAD ${ }^{+}$-dependent Sir2p histone deacetylase is a negative regulator of chromosomal DNA replication. Genes Dev 18: 769-781.

Polevoda B, Sherman F. 2003. Composition and function of the eukaryotic $\mathrm{N}$-terminal acetyltransferase subunits. Biochem Biophys Res Commun 308: 1-11.

Rampakakis E, Arvanitis DN, Di Paola D, Zannis-Hadjopoulos M. 2009. Metazoan origins of DNA replication: Regulation through dynamic chromatin structure. J Cell Biochem 106: 512-520.

Rao H, Stillman B. 1995. The origin recognition complex interacts with a bipartite DNA binding site within yeast replicators. Proc Natl Acad Sci 92: 2224-2228.

Remus D, Beall EL, Botchan MR. 2004. DNA topology, not DNA sequence, is a critical determinant for Drosophila ORC-DNA binding. EMBO $J$ 23: 897-907.

Ross PL, Huang YN, Marchese JN, Williamson B, Parker K, Hattan S, Khainovski N, Pillai S, Dey S, Daniels S, et al. 2004. Multiplexed protein quantitation in Saccharomyces cerevisiae using amine-reactive isobaric tagging reagents. Mol Cell Proteomics 3: 1154-1169.

Sampath V, Yuan P, Wang IX, Prugar E, van Leeuwen F, Sternglanz R. 2009. Mutational analysis of the Sir3 BAH domain reveals multiple points of interaction with nucleosomes. Mol Cell Biol 29: 2532-2545.

Sclafani RA, Holzen TM. 2007. Cell cycle regulation of DNA replication. Annu Rev Genet 41: 237-280.

Shimada K, Pasero P, Gasser SM. 2002. ORC and the intraS-phase checkpoint: A threshold regulates Rad53p activation in S phase. Genes Dev 16: 3236-3252.

Shor E, Warren CL, Tietjen J, Hou Z, Muller U, Alborelli I, Gohard FH, Yemm AI, Borisov L, Broach JR, et al. 2009. The origin recognition complex interacts with a subset of metabolic genes tightly linked to origins of replication. PLOS Genet 5: e1000755. doi: 10.1371/journal.pgen.1000755.

Simpson RT. 1990. Nucleosome positioning can affect the function of a cis-acting DNA element in vivo. Nature 343: 387-389.

Stillman B. 2005. Origin recognition and the chromosome cycle. FEBS Lett 579: 877-884.

Suter B, Tong A, Chang M, Yu L, Brown GW, Boone C, Rine J. 2004. The origin recognition complex links replication, sister chromatid cohesion and transcriptional silencing in Saccharomyces cerevisiae. Genetics 167: 579-591.
Vashee S, Cvetic C, Lu W, Simancek P, Kelly TJ, Walter JC. 2003. Sequence-independent DNA binding and replication initiation by the human origin recognition complex. Genes Dev 17: 1894-1908.

Vogelauer M, Rubbi L, Lucas I, Brewer BJ, Grunstein M. 2002. Histone acetylation regulates the time of replication origin firing. Mol Cell 10: 1223-1233.

Wang X, Connelly JJ, Wang CL, Sternglanz R. 2004. Importance of the Sir3 $\mathrm{N}$ terminus and its acetylation for yeast transcriptional silencing. Genetics 168: 547-551.

Wilmes GM, Bell SP. 2002. The B2 element of the Saccharomyces cerevisiae ARS1 origin of replication requires specific sequences to facilitate pre-RC formation. Proc Natl Acad Sci 99: 101-106.

Wu PY, Nurse P. 2009. Establishing the program of origin firing during $S$ phase in fission yeast. Cell 136: 852-864.

Xu W, Aparicio JG, Aparicio OM, Tavare S. 2006. Genome-wide mapping of ORC and $\mathrm{Mcm} 2 \mathrm{p}$ binding sites on tiling arrays and identification of essential ARS consensus sequences in $S$. cerevisiae. BMC Genomics 7: 276. doi: 10.1186/1471-21647-276.

Yan W, Craig EA. 1999. The glycine-phenylalanine-rich region determines the specificity of the yeast Hsp40 Sis1. Mol Cell Biol 19: 7751-7758.

Zhang Z, Hayashi MK, Merkel O, Stillman B, Xu RM. 2002. Structure and function of the BAH-containing domain of Orclp in epigenetic silencing. EMBO J 21: 4600-4611. 


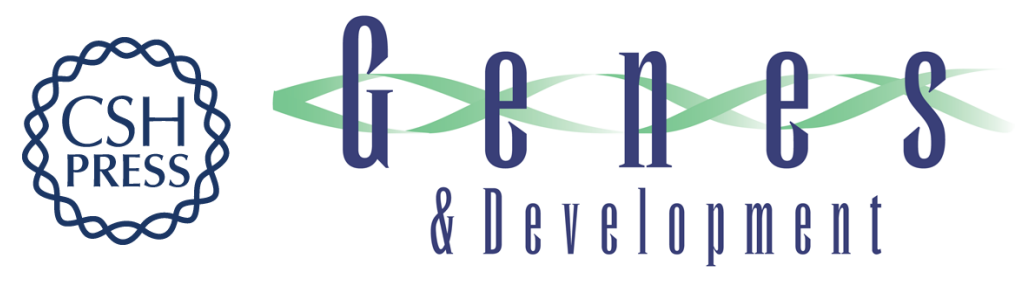

\section{The conserved bromo-adjacent homology domain of yeast Orc1 functions in the selection of DNA replication origins within chromatin}

Philipp Müller, Sookhee Park, Erika Shor, et al.

Genes Dev. 2010, 24:

Access the most recent version at doi:10.1101/gad.1906410

Supplemental
Material http://genesdev.cshlp.org/content/suppl/2010/06/25/24.13.1418.DC1

References This article cites 67 articles, 34 of which can be accessed free at:

http://genesdev.cshlp.org/content/24/13/1418.full.html\#ref-list-1

License

Email Alerting Receive free email alerts when new articles cite this article - sign up in the box at the top

Service

right corner of the article or click here.

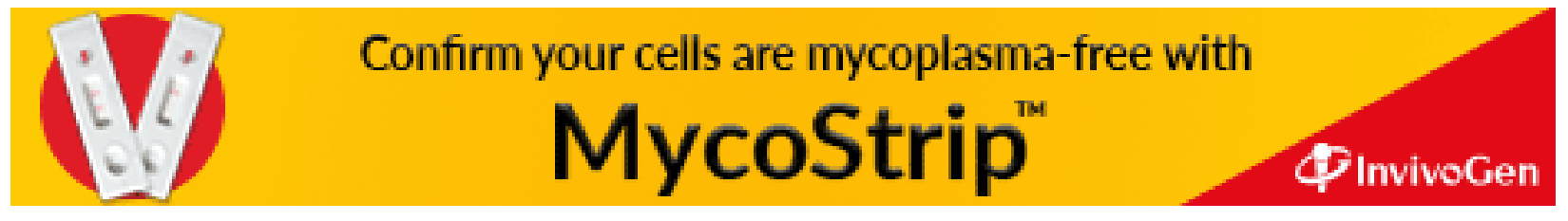

